\title{
The landscape of host genetic factors involved in immune response to common viral infections
}

Linda Kachuri ${ }^{1 \dagger}$, Stephen S. Francis ${ }^{1,2,3,4^{*}+}{ }$, Maike L. Morrison ${ }^{5,6,7}$, George A. Wendt ${ }^{2}$, Yohan Bossé ${ }^{8}$, Taylor B. Cavazos ${ }^{9}$, Sara R. Rashkin ${ }^{1,10}$, Elad Ziv ${ }^{3,11,12}$ and John S. Witte $1,3,5,12,13^{*}$

\begin{abstract}
Background: Humans and viruses have co-evolved for millennia resulting in a complex host genetic architecture. Understanding the genetic mechanisms of immune response to viral infection provides insight into disease etiology and therapeutic opportunities.

Methods: We conducted a comprehensive study including genome-wide and transcriptome-wide association analyses to identify genetic loci associated with immunoglobulin $\mathrm{G}$ antibody response to 28 antigens for 16 viruses using serological data from 7924 European ancestry participants in the UK Biobank cohort.

Results: Signals in human leukocyte antigen (HLA) class II region dominated the landscape of viral antibody response, with 40 independent loci and 14 independent classical alleles, 7 of which exhibited pleiotropic effects across viral families. We identified specific amino acid (AA) residues that are associated with seroreactivity, the strongest associations presented in a range of AA positions within DRß1 at positions 11, 13, 71, and 74 for EpsteinBarr virus (EBV), Varicella zoster virus (VZV), human herpesvirus 7, (HHV7), and Merkel cell polyomavirus (MCV). Genome-wide association analyses discovered 7 novel genetic loci outside the HLA associated with viral antibody response $\left(P<5.0 \times 10^{-8}\right)$, including FUT2 (19q13.33) for human polyomavirus BK (BKV), STING1 (5q31.2) for MCV, and CXCR5 (11q23.3) and TBKBP1 (17q21.32) for HHV7. Transcriptome-wide association analyses identified 114 genes associated with response to viral infection, 12 outside of the HLA region, including ECSCR: $P=5.0 \times 10^{-15}$ (MCV), NTN5: $P=1.1 \times 10^{-9}(\mathrm{BKV})$, and $P 2 R Y 13: P=1.1 \times 10^{-8}$ EBV nuclear antigen. We also demonstrated pleiotropy between viral response genes and complex diseases, from autoimmune disorders to cancer to neurodegenerative and psychiatric conditions.
\end{abstract}

Conclusions: Our study confirms the importance of the HLA region in host response to viral infection and elucidates novel genetic determinants beyond the HLA that contribute to host-virus interaction.

Keywords: Infection, Virus, Serology, Antigen, Antibody, Immunoglobulin G, Immune response, Human leukocyte antigen (HLA), Polyomavirus, Genome-wide association study (GWAS), Transcriptome-wide association study (TWAS)

\footnotetext{
* Correspondence: stephen.francis@ucsf.edu; jwitte@ucsf.edu

${ }^{\dagger}$ Linda Kachuri and Stephen S. Francis contributed equally to this work.

1 Department of Epidemiology and Biostatistics, University of California San Francisco, San Francisco, CA, USA

Full list of author information is available at the end of the article
}

(c) The Author(s). 2020 Open Access This article is licensed under a Creative Commons Attribution 4.0 International License, which permits use, sharing, adaptation, distribution and reproduction in any medium or format, as long as you give appropriate credit to the original author(s) and the source, provide a link to the Creative Commons licence, and indicate if changes were made. The images or other third party material in this article are included in the article's Creative Commons licence, unless indicated otherwise in a credit line to the material. If material is not included in the article's Creative Commons licence and your intended use is not permitted by statutory regulation or exceeds the permitted use, you will need to obtain permission directly from the copyright holder. To view a copy of this licence, visit http://creativecommons.org/licenses/by/4.0/ The Creative Commons Public Domain Dedication waiver (http://creativecommons.org/publicdomain/zero/1.0/) applies to the data made available in this article, unless otherwise stated in a credit line to the data. 


\section{Background}

Viruses have been infecting cells for a half a billion years [1]. During our extensive co-evolution, viruses have exerted significant selective pressure on humans and vice versa, overtly during fatal outbreaks, and covertly through cryptic immune interaction when a pathogen remains latent. The recent pandemic of severe acute respiratory syndrome coronavirus 2 (SARS-CoV-2) highlights the paramount public health need to understand human genetic variation in response to viral challenge. Clinical variation in COVID-19 severity and symptomatic presentation may be due to differences host genetic factors relating to immune response [2]. Furthermore, many common infections are cryptically associated with a variety of complex illnesses, especially those with an immunologic component, from cancer to autoimmune and neurologic conditions [3-5]. Despite their broad health relevance, few large-scale genome-wide association studies (GWAS) have been conducted on serological response phenotypes [6-10]. Understanding the genetic architecture of immunologic response to viruses may therefore provide new insight into etiologic mechanisms of diverse complex diseases.

Several common viruses exert a robust cell-mediated and humoral immune response that bi-directionally modulates the balance between latent and lytic infection. Studies have demonstrated a strong heritable component (32-48\%) of antibody response [11] and identified associations between host polymorphisms in genes relating to cell entry, cytokine production, and immune response and a variety of viruses [12]. The predominance of previously reported associations has implicated genetic variants in human leucocyte antigen (HLA) class I and II genes in the modulation of immune response to diverse viral antigens $[7,13]$.

In this study, we utilize data from the UK Biobank (UKB) cohort [14] to evaluate the relationship between host genetics and immunoglobulin $\mathrm{G}$ antibody response to 28 antigens for 16 viruses. Immunoglobulin G (IgG) antibody is the most common antibody in blood, which serves as a stable biomarker of lifetime exposure to common viruses. High levels of specific IgG's can be the result of chronic infection, while low levels may indicate poor immunity. Viruses assayed in the UKB multiplex serology panel were previously chosen based on putative links to chronic diseases including cancer, autoimmune, and neurodegenerative conditions [15]. We conduct integrative genome-wide and transcriptome-wide analyses of antibody response and positivity to viral antigens (Fig. 1), which elucidate novel genetic underpinnings of viral infection and immune response.

\section{Methods}

\section{Study population and phenotypes}

The UK Biobank (UKB) is a population-based prospective cohort of over 500,000 individuals aged $40-69$ years at enrollment in 2006-2010 who completed extensive questionnaires and physical assessments and provided blood samples [14]. Analyses were restricted to individuals of predominantly European ancestry based on selfreport and after excluding samples with any of the first two genetic ancestry principal components (PCs) outside of 5 standard deviations (SD) of the population mean (Additional file 1: Figure S1). We removed samples with discordant self-reported and genetic sex, samples with call rates $<97 \%$ or heterozygosity $>5$ SD from the mean, and one sample from each pair of first-degree relatives identified using KING [16].

Of the 413,810 European ancestry individuals available for analysis, a total of 7948 had serological measures. A multiplex serology panel (IgG) was performed over a 2week period using previously developed methods $[17,18]$ that have been successfully applied in epidemiological studies [7, 19]. Details of the serology methods and assay validation performance are described in Mentzer et al. [15] Briefly, multiplex serology was performed using a bead-based glutathione S-transferase (GST) capture assay with glutathione-casein coated fluorescence-labeled polystyrene beads and pathogen-specific GST-X-tag fusion proteins as antigens [15]. Each antigen was loaded onto a distinct bead set and the beads were simultaneously presented to primary serum antibodies at serum dilution 1: 1000 [15]. Immunocomplexes were quantified using a Luminex 200 flow cytometer, which produced Median Fluorescence Intensities (MFI) for each antigen. The serology assay showed adequate performance, with a median coefficient of variation (CV) of $17 \%$ across all antigens and $3.5 \%$ among seropositive samples only [15].

\section{Genome-wide association analysis}

We evaluated the relationship between genetic variants across the genome and serological phenotypes using PLINK 2.0 (October 2017 version) [20]. Participants were genotyped on the Affymetrix Axiom UK Biobank array (89\%) or the UK BiLEVE array (11\%) [14] with genome-wide imputation performed using the Haplotype Reference Consortium data and the merged UK10K and 1000 Genomes phase 3 reference panels [14]. We excluded variants out of Hardy-Weinberg equilibrium at $p<1 \times 10^{-5}$, call rate $<95 \%$ (alternate allele dosage within 0.1 of the nearest hard call to be non-missing), imputation quality INFO $<0.30$, and $\mathrm{MAF}<0.01$.

Seropositivity for each antigen was determined using established cut-offs based on prior validation work [15]. The primary GWAS focused on continuous phenotypes (MFI values), which measure the magnitude of antibody response, also referred to as seroreactivity. These analyses were conducted among seropositive individuals only for antigens with seroprevalence of $\geq 20 \%$ ( $n=$ 1500 ) based on $80 \%$ power to detect only common 


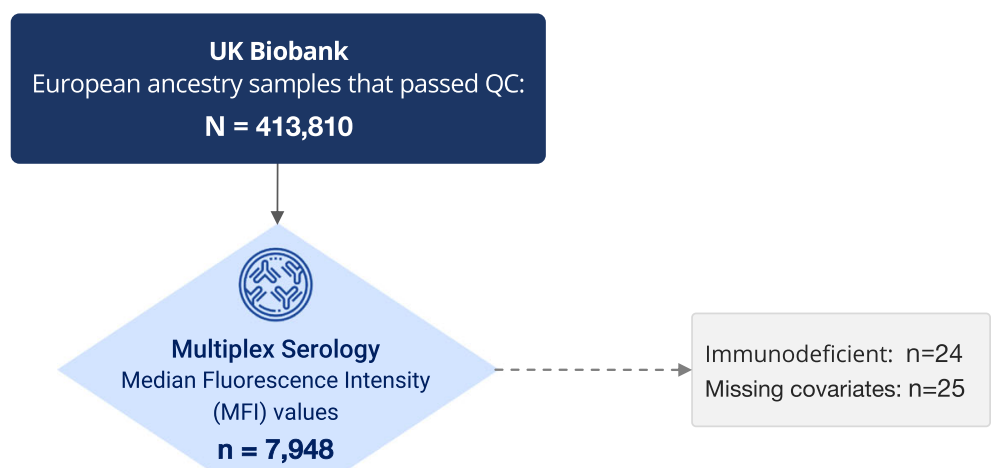

$\mathrm{n}=7,948$

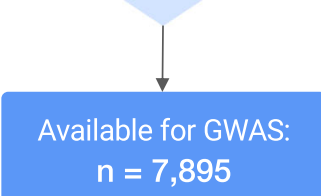

Serostatus

Binary Phenotypes

Determined based on established cutoffs for seropositivity for each antigen

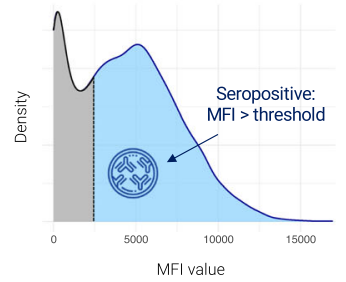

Antigens with $\geq 100$ cases ( + or -)

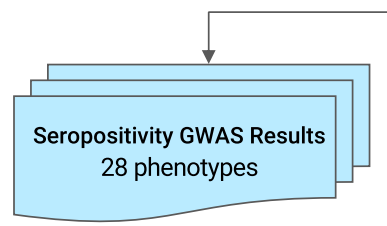

Seroreactivity

Quantitative Phenotypes

Magnitude of antibody response (IgG)

in seropositive individuals only

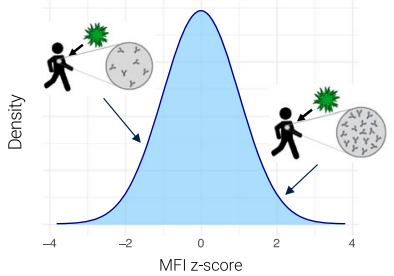

Antigens with $\geq 20 \%$ seropositivity

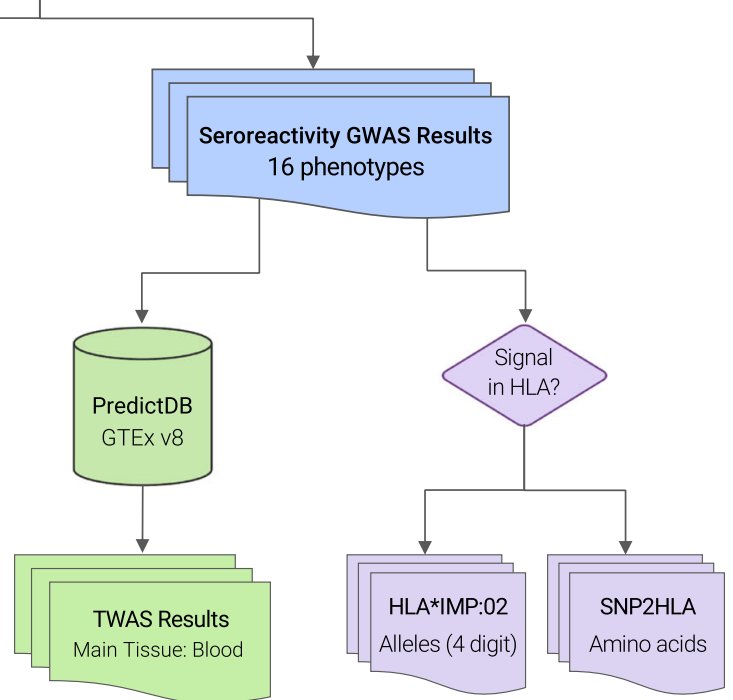

Fig. 1 Flow chart describing the main serological phenotypes and association analyses

variants with large effect sizes at this sample size (Additional file 1: Figure S2). MFI values were transformed to standardized, normally distributed $z$-scores using ordered quantile normalization [21].

Seroreactivity GWAS was conducted using linear regression with adjustment for age at enrollment, sex, body mass index (BMI), socioeconomic status (Townsend deprivation index), the presence of any autoimmune and/ or inflammatory conditions, genotyping array, serology assay date, quality control flag indicating sample spillover or an extra freeze/thaw cycle, and the top 10 genetic ancestry principal components (PC's). Autoimmune and 
chronic inflammatory conditions were identified using the following primary and secondary diagnostic ICD-10 codes (E10, M00-03, M05-M14, M32, L20-L30, L40, G35, K5052, K58, G61) in Hospital Episode Statistics. Individuals diagnosed with any immunodeficiency (ICD-10 D80-89, $n=24$ ) were excluded from all analyses.

For all antigens with at least 100 seropositive (or seronegative for pathogens with ubiquitous exposure) individuals, GWAS of discrete seropositivity phenotypes was undertaken using logistic regression, adjusting for the same covariates listed above.

The functional relevance of the lead GWAS loci for antibody response was assessed using in silico functional annotation analyses based on Combined Annotation Dependent Depletion (CADD) [22] scores and RegulomeDB 2.0 [23] and by leveraging external datasets, such as GTEx v8, DICE (Database of Immune Cell Expression) [24], and the Human Plasma Proteome Atlas [25, 26].

\section{Cross-trait associations with disease}

We explored pleiotropic associations between lead variants influencing antibody levels and several chronic diseases with known or hypothesized viral risk factors. Associations with selected cancers were obtained from a cancer pleiotropy meta-analysis of the UK Biobank and Genetic Epidemiology Research on Aging cohorts [27]. Summary statistics for the schizophrenia GWAS of 33, 640 cases and 43,456 controls by Lam et al. [28] were downloaded from the Psychiatric Genomics Consortium. Association $p$ values were obtained from the National Institute on Aging Genetics of Alzheimer's Disease Data Storage Site for the GWAS by Jun et al. [29], which included 17,536 cases and 53,711 controls. Associations with $p<7.3 \times 10^{-4}$ were considered statistically significant after correction for the number of variants and phenotypes tested.

\section{HLA regional analysis}

For phenotypes displaying a genome-wide significant signal in the HLA region, independent association signals were ascertained using two complementary approaches: clumping and conditional analysis. Clumping is a postprocessing step applied to GWAS summary statistics to identify independent association signals by grouping variants based on LD within specific windows. Clumping was performed on all variants with $P<5 \times 10^{-8}$ for each phenotype, as well as across phenotypes. Clumps were formed around index variants with the lowest $p$ value, and all other variants with LD $r^{2}>0.05$ within a \pm 500 $\mathrm{kb}$ window were considered non-independent and assigned to that variant's clump.

Next, we conducted conditional analyses using a forward stepwise strategy to identify statistically independent signals within each type of variant (SNP/indel or classical HLA allele). Unlike clumping, conditional analyses involve fitting a new model that includes specific variants as covariates, thereby directly accounting for LD and providing association estimates that are adjusted for other relevant SNP effects. A total of 38,655 SNPs/indels on chromosome $6(29,600,000-33,200,000 \mathrm{bp})$ were extracted to conduct regional analyses. Classical HLA alleles were imputed for UKB participants at 4-digit resolution using the HLA*IMP:02 algorithm [14], with modified settings to accommodate the addition of diverse samples from population reference panels described by Motyer et al. [30]. Details of the HLA imputation procedure are described in UKB Resource 182. Imputed dosages were available for 362 classical alleles in 11 genes: HLA-A, HLA-B, and HLA-C (class I); HLADRB5, HLA-DRB4, HLA-DRB3, HLA-DRB1, HLA-DQA1, $H L A-D Q B 1, H L A-D P A 1$, and HLA-DPB1 (class II). Allele names with "99:01" for DRB3/4/5, which denote copy number absence, were renamed as "00:00" to avoid confusion with traditional HLA nomenclature. We also used SNP2HLA [31] to impute HLA alleles and corresponding amino acid sequences at a 4-digit resolution in $H L A$ A, HLA-B, HLA-C, HLA-DRB1, HLA-DQA1, HLA-DQB1, $H L A-D P A 1$, and $H L A-D P B 1$ using the Type 1 Diabetes Genetics Consortium (T1DGC) reference panel comprised of 2767 unrelated individuals of European descent. T1DGC was also among several reference datasets used by HLA*IMP:02. SNP2HLA imputation was conducted using 100-kb windows.

Analyses were restricted to common HLA alleles and amino acid sequences (frequency $\geq 0.01$ ) with imputation quality scores $>0.30$, for a total of 1081 markers (101 alleles + 980 amino acid residues). We performed uncertaintyaware analyses using the imputed allele dosages, which is preferred to hard-thresholding approaches [32]. Linear regression models were adjusted for the same set of covariates as the GWAS. Associations for each marker were considered statistically significant if $P<4.6 \times 10^{-5}$ based on Bonferroni correction for 1081 tests.

For each antigen response phenotype, we identified SNPs/indels or classical HLA alleles with the lowest $p$ value, among variants that achieved Bonferronisignificant associations $\left(P<4.6 \times 10^{-5}\right)$, and performed forward iterative conditional regression to identify other independent signals, until no associations with a conditional $p$ value $\left(P_{\text {cond }}\right)<5 \times 10^{-8}$ remained. We also assessed the independence of associations across different types of genetic variants by including conditionally independent HLA alleles as covariates in the SNP-based analysis.

For amino acid positions with $>2$ possible residues (alleles), we applied the haplotype omnibus test in PLINK 1.07 [33] to obtain an overall $p$ value for jointly testing all possible substitutions at that specific position. The 
omnibus test was applied to all amino acid residues at a given position, even if not all substitutions achieved the Bonferroni-corrected threshold $\left(P<4.6 \times 10^{-5}\right)$ in the single-marker analysis. The frequency of amino acid substitutions at specific HLA alleles was determined using European ancestry reference populations part of the Allele Frequency Net Database (AFND 2020) [34].

\section{Transcriptome-wide association analysis}

Gene transcription levels were imputed and analyzed using the MetaXcan approach [35] applied to GWAS summary statistics. For imputation, we used biologically informed MASHR-M prediction models [36] obtained from the PredictDB repository [37]. These models are based on GTEx v8 with effect sizes computed using MASHR (Multivariate Adaptive Shrinkage in R) [38] for variants fine-mapped with DAP-G (Deterministic Approximation of Posteriors) [39, 40]. An advantage of this approach is that MASHR effect sizes are smoothed by taking advantage of the correlation in cis-eQTL effects across tissues. For each antigen, we performed a transcriptome-wide association study (TWAS) using gene expression levels in whole blood. Statistically significant associations for each gene were determined based on the Bonferroni correction for the number of genes tested.

We also examined gene expression profiles in tissues that represent known infection targets or related pathologies. Human herpesviruses and polyomaviruses are neurotropic and have been implicated in several neurological conditions [41, 42]; therefore, we considered gene expression in the frontal cortex. For Epstein-Barr virus (EBV) antigens additional models included EBVtransformed lymphocytes. Merkel cell polyomavirus (MCV) is a known cause of Merkel cell carcinoma [43], a rare but aggressive type of skin cancer; therefore, we examined transcriptomic profiles in skin tissues for MCV only.

Pathways represented by genes associated with antibody response to viral antigens were summarized by conducting enrichment analysis based on curated gene sets using the $\mathrm{R}$ package clusterProfiler (version 3.12.0) [44]. Significantly associated TWAS genes were grouped by virus family (herpesviruses vs. polyomaviruses) and specificity of association (multiple antigens vs. single antigen).

\section{Results}

A random sample of the participants representative of the full UKB cohort was assayed using a multiplex serology panel [15]. We analyzed data from 7924 participants of predominantly European ancestry, described in (Additional file 2: Table S1). Approximately 90\% of individuals were seropositive for herpes family viruses with ubiquitous exposure: EBV (EBV EA-D 86.2\% to ZEBRA 91.2\%), human herpesvirus 7 (HHV7 94.8\%), and varicella zoster virus (VZV 92.3\%). Seroprevalence was somewhat lower for cytomegalovirus (CMV), ranging between 56.5\% (CMV pp28) and 63.3\% (CMV pp52), and herpes simplex virus-1 (HSV1 69.3\%). Human polyomavirus BKV was more prevalent (95.3\%) compared to other polyomaviruses, Merkel cell polyomavirus (MCV $66.1 \%$ ) and polyomavirus JC (JCV) (56.6\%). Less common infections included HSV-2 (15.2\%), HPV16 (E6 and E7 oncoproteins: 4.7\%), HPV18 (2.4\%), human T-cell lymphotropic virus type 1 (HTLV1, 1.6\%), hepatitis B (HBV, 1.6\%), and hepatitis C (HCV, 0.3\%).

\section{Genetic determinants of response to viral infection}

Results from our GWAS of antibody response phenotypes were dominated by signals in the HLA region, which were detected for all EBV antigens (EA-D, EBNA, p18, ZEBRA), CMV pp52, HSV1, HHV7, VZV, JCV, and MCV (Table 1; Additional file 1: Figure S3). Most of the top-ranking HLA variants for each antigen were independent of those for other antigens based on $r^{2}$ but not $D^{\prime}$ (Additional file 1: Figure S4). Exceptions were moderate LD between lead variants for EBV ZEBRA and HSV1 $\left(r^{2}=0.45\right)$, EBV EBNA and JCV $\left(r^{2}=0.45\right)$, and HHV7 and MCV $\left(r^{2}=0.44\right)$. However, based on the complex LD structure and effect sizes, we cannot rule out that these linked to rare haplotypes. Outside of the HLA region, genome-wide significant associations with seroreactivity were detected for $\mathrm{MCV}$ at $3 \mathrm{p} 24.3$ (rs776170649, LOC339862: $P=1.7 \times 10^{-8}$ ) and 5q31.2 (rs7444313, TMEM173 (also known as STING1): $P=$ $2.4 \times 10^{-15}$ ); BKV at 19q13.3 (rs681343, FUT2: $P=4.7 \times$ $10^{-15}$ ) (Fig. 2); EBV EBNA at 3q25.1 (rs67886110, MED12L: $P=1.3 \times 10^{-9}$ ); HHV-7 at 11q23.3 (rs75438046, CXCR5: $\left.P=1.3 \times 10^{-8}\right)$ and $17 \mathrm{q} 21.3(\mathrm{rs} 1808192$, TBKBP1: $\left.P=9.8 \times 10^{-9}\right)$; and HSV-1 at 10q23.3 (rs11203123: $P=$ $3.9 \times 10^{-8}$ ). However, the loci outside of HLA identified for HHV7 and HSV1 were not statistically significant considering a more stringent significance threshold corrected for the number of seroreactivity phenotypes tested $(P<$ $\left.5.0 \times 10^{-8} / 16=3.1 \times 10^{-9}\right)$.

GWAS of discrete seropositivity phenotypes identified associations in HLA for EBV EA-D (rs2395192: OR = 0.66, $P=4.0 \times 10^{-19}$ ), EBV EBNA (rs9268848: OR $=1.60$, $P=1.2 \times 10^{-18}$ ), EBV ZEBRA (rs17211342: $0.63, P=$ $\left.1.6 \times 10^{-15}\right)$, VZV $\left(\mathrm{rs} 3096688: \mathrm{OR}=0.70, P=3.7 \times 10^{-8}\right)$, JCV (rs9271147: OR $=0.54, P=1.3 \times 10^{-42}$ ), and $\mathrm{MCV}$ (rs17613347: $\mathrm{OR}=0.61, P=1.2 \times 10^{-26}$ ) (Additional file 1: Figure S3; Additional file 2: Table S2). An association with susceptibility to MCV infection was also observed at 5q31.2 (5:138845045_TTATC_T, ECSCR: OR = 1.26, $P=$ $\left.7.2 \times 10^{-9}\right)$, with high LD $\left(r^{2}=0.95\right)$ between seroreactivity and seropositivity lead variants. 
Table 1 Lead genome-wide significant variants $\left(P<5.0 \times 10^{-8}\right)$ for continuous antibody response phenotypes for antigens with at least 20\% seroprevalence

\begin{tabular}{|c|c|c|c|c|c|c|c|c|c|c|c|c|c|}
\hline \multicolumn{2}{|c|}{ Antigen } & \multirow{2}{*}{$\begin{array}{l}N \\
5000\end{array}$} & \multirow{2}{*}{$\begin{array}{l}\text { Chr } \\
6\end{array}$} & \multirow{2}{*}{$\begin{array}{l}\text { Position } \\
32301427\end{array}$} & \multirow{2}{*}{$\begin{array}{l}\text { Variant } \\
\text { rs115378818 }\end{array}$} & \multicolumn{2}{|l|}{ Alleles } & \multirow{2}{*}{$\begin{array}{l}\text { EAF } \\
0.978\end{array}$} & \multirow{2}{*}{$\begin{array}{l}\text { Beta }^{2} \\
0.633\end{array}$} & \multirow{2}{*}{$\begin{array}{l}\text { (SE) } \\
(0.095)\end{array}$} & \multirow{2}{*}{$\begin{array}{l}\mathbf{P} \\
2.9 \times 10^{-11}\end{array}$} & \multirow{2}{*}{$\begin{array}{l}\text { Function } \\
\text { intronic }\end{array}$} & \multirow{2}{*}{$\begin{array}{l}\text { Nearest gene } \\
T S B P 1\end{array}$} \\
\hline CMV & pp52 & & & & & $\begin{array}{l}\text { Effect } \\
C\end{array}$ & $\begin{array}{l}\text { Other } \\
T\end{array}$ & & & & & & \\
\hline EBV & EA-D & 6806 & 6 & 32665840 & rs34825357 & $\mathrm{T}$ & $\mathrm{TC}$ & 0.409 & -0.114 & $(0.017)$ & $2.0 \times 10^{-11}$ & intergenic & МТСОЗР1 \\
\hline \multirow[t]{2}{*}{ EBV } & EBNA & 7003 & 3 & 151114852 & rs67886110* & G & $\mathrm{T}$ & 0.596 & 0.103 & $(0.017)$ & $1.3 \times 10^{-9}$ & intronic & MED12L \\
\hline & & & 6 & 32451762 & rs9269233 & A & C & 0.249 & 0.315 & (0.019) & $3.5 \times 10^{-61}$ & intergenic & HLA-DRB9 \\
\hline EBV & VCA p18 & 7492 & 6 & 31486158 & $6: 31486158$ & GT & G & 0.245 & 0.197 & $(0.018)$ & $7.1 \times 10^{-27}$ & intergenic & PPIAPG \\
\hline EBV & ZEBRA & 7197 & 6 & 32637772 & rs9274728 & A & G & 0.718 & -0.315 & (0.018) & $4.7 \times 10^{-67}$ & intergenic & HLA-DQB1 \\
\hline HHV6 & IE1A & 6077 & 7 & 139985625 & rs2429218 & $\mathrm{T}$ & $C$ & 0.615 & 0.106 & (0.019) & $1.4 \times 10^{-8}$ & downstream & RP5-1136G2.1 \\
\hline \multirow[t]{3}{*}{ HHV7 } & U14 & 7481 & 6 & 32602665 & rs139299944 & C & CT & 0.655 & 0.114 & $(0.017)$ & $1.5 \times 10^{-11}$ & intronic & HLA-DQA1 \\
\hline & & & 11 & 118767564 & rs75438046 & G & A & 0.970 & 0.280 & $(0.049)$ & $1.3 \times 10^{-8}$ & 3'-UTR & CXCR5 / BCL9L \\
\hline & & & 17 & 45794706 & rs1808192 & A & G & 0.331 & -0.099 & $(0.017)$ & $9.8 \times 10^{-9}$ & intergenic & TBKBP1 \\
\hline \multirow[t]{2}{*}{ HSV1 } & $1 g G$ & 5468 & 6 & 32627852 & rs1130420 & G & A & 0.583 & -0.122 & (0.019) & $2.5 \times 10^{-10}$ & 3'-UTR & $H L A-D Q B 1$ \\
\hline & & & 10 & 91189187 & rs11203123* & $A$ & $C$ & 0.988 & 0.512 & (0.093) & $3.9 \times 10^{-8}$ & intergenic & SLC16A12 \\
\hline VZV & $\mathrm{gE} / \mathrm{Ig}^{1}$ & 7289 & 6 & 32623193 & rs9273325 & G & A & 0.831 & -0.232 & $(0.021)$ & $8.2 \times 10^{-28}$ & intergenic & $H L A-D Q B 1$ \\
\hline BKV & VP1 & 7523 & 19 & 49206462 & rs681343 & $C$ & T & 0.491 & -0.125 & $(0.016)$ & $4.7 \times 10^{-15}$ & synonymous & FUT2 \\
\hline $\mathrm{JCV}$ & VP1 & 4471 & 6 & 32589842 & rs9271525 & G & A & 0.163 & -0.318 & $(0.031)$ & $3.9 \times 10^{-24}$ & intergenic & HLA-DQA1 \\
\hline \multirow[t]{3}{*}{ MCV } & VP1 & 5219 & 3 & 18238783 & rs776170649 & $C T$ & $C$ & 0.790 & -0.134 & $(0.024)$ & $1.7 \times 10^{-8}$ & intergenic & LOC339862 \\
\hline & & & 5 & 138865423 & rs7444313 & G & A & 0.263 & 0.169 & $(0.021)$ & $2.4 \times 10^{-15}$ & intergenic & TMEM173 \\
\hline & & & 6 & 32429277 & rs9268847 & A & G & 0.750 & -0.195 & $(0.022)$ & $2.4 \times 10^{-19}$ & intronic & HLA-DRB9 \\
\hline
\end{tabular}

${ }^{1} \mathrm{VZV}$ antigens $\mathrm{gE}$ and $\mathrm{gl}$ were co-loaded onto the same Luminex bead set

${ }^{2}$ Regression coefficients were estimated per 1 standard deviation increase in normalized MFI value $z$-scores with adjustment for age at enrollment, sex, body mass index, socioeconomic status (Townsend deprivation index), the presence of any autoimmune conditions, genotyping array, serology assay date, quality control flag, and the top 10 genetic ancestry principal components

*Multi-allelic variants: rs67886110 (G/T and G/C) and rs11203123 (A/C and A/AC)

Several genome-wide significant associations were observed for antigens with $<20 \%$ seroprevalence, which were not included in the GWAS of antibody response due to inadequate sample size (Additional file 2: Table S2). Infection susceptibility variants were identified for HSV2 in 17p13.2 (rs2116443: OR=1.28, $P=4.5 \times 10^{-8}$; ITGAE); HPV16 E6 and E7 oncoproteins in $6 \mathrm{p} 21.32$ (rs601148: $\left.\mathrm{OR}=0.60, P=3.3 \times 10^{-9} ; H L A-D R B 1\right)$ and 19q12 (rs144341759: OR=0.383, $P=4.0 \times 10^{-8}$; CTC448F2.6); and HPV18 in 14q24.3 (rs4243652: OR = 3.13, $P=7.0 \times 10^{-10}$ ). Associations were also detected for Kaposi's sarcoma-associated herpesvirus (KSHV), HTLV1, $\mathrm{HBV}$, and $\mathrm{HCV}$, including a variant in the MERTK oncogene (HCV Core rs199913364: OR $=0.25, P=1.2 \times 10^{-8}$ ). After correcting for 28 serostatus phenotypes tested $(P<$ $1.8 \times 10^{-9}$ ), the only statistically significant associations remained for EBV EA-D (rs2395192), EBV EBNA (rs9268848), EBV ZEBRA (rs17211342), JCV (rs9271147), MCV (rs17613347), and HPV18 (rs4243652).

\section{Functional characterization of GWAS findings}

In silico functional analyses of the lead 17 GWAS variants identified enrichment for multiple regulatory elements (summarized in Additional file 2: Table S3).
Three variants were predicted to be in the top $10 \%$ of deleterious substitutions in GRCh37 based on CADD scores >10: $\quad$ rs776170649 (MCV, $\mathrm{CADD}=15.61)$, rs139299944 (HHV7, CADD = 12.15), and rs9271525 $(\mathrm{JCV}, \mathrm{CADD}=10.73)$. Another HHV7-associated variant, rs1808192 (RegulomeDB rank: 1f), an eQTL and sQTL for TBKBP1, mapped to 44 functional elements for multiple transcription factors, including IKZF1, a critical regulator of lymphoid differentiation frequently mutated in B cell malignancies.

Eleven sentinel variants were eQTLs and 8 were splicing QTLs in GTEx, with significant (FDR < 0.05) effects across multiple genes and tissues (Additional file 1: Figure S5). The most common eQTL and sQTL targets included HLA-DQA1, HLA-DQA2, HLA-DQB1, HLA-DQB2, HLADRB1, and HLA-DRB6. Outside of HLA, rs681343 (BKV), a synonymous FUT2 variant was an eQTL for 8 genes, including FUT2 and NTN5. MCV variant in 5q31.2, rs7444313, was an eQTL for 7 genes, with concurrent sQTL effects on TMEM173, also known as STING1 (stimulator of interferon response cGAMP interactor 1) and CXXC5. Gene expression profiles in immune cell populations from DICE [24] identified several cell-typespecific effects that were not observed in GTEx. An 

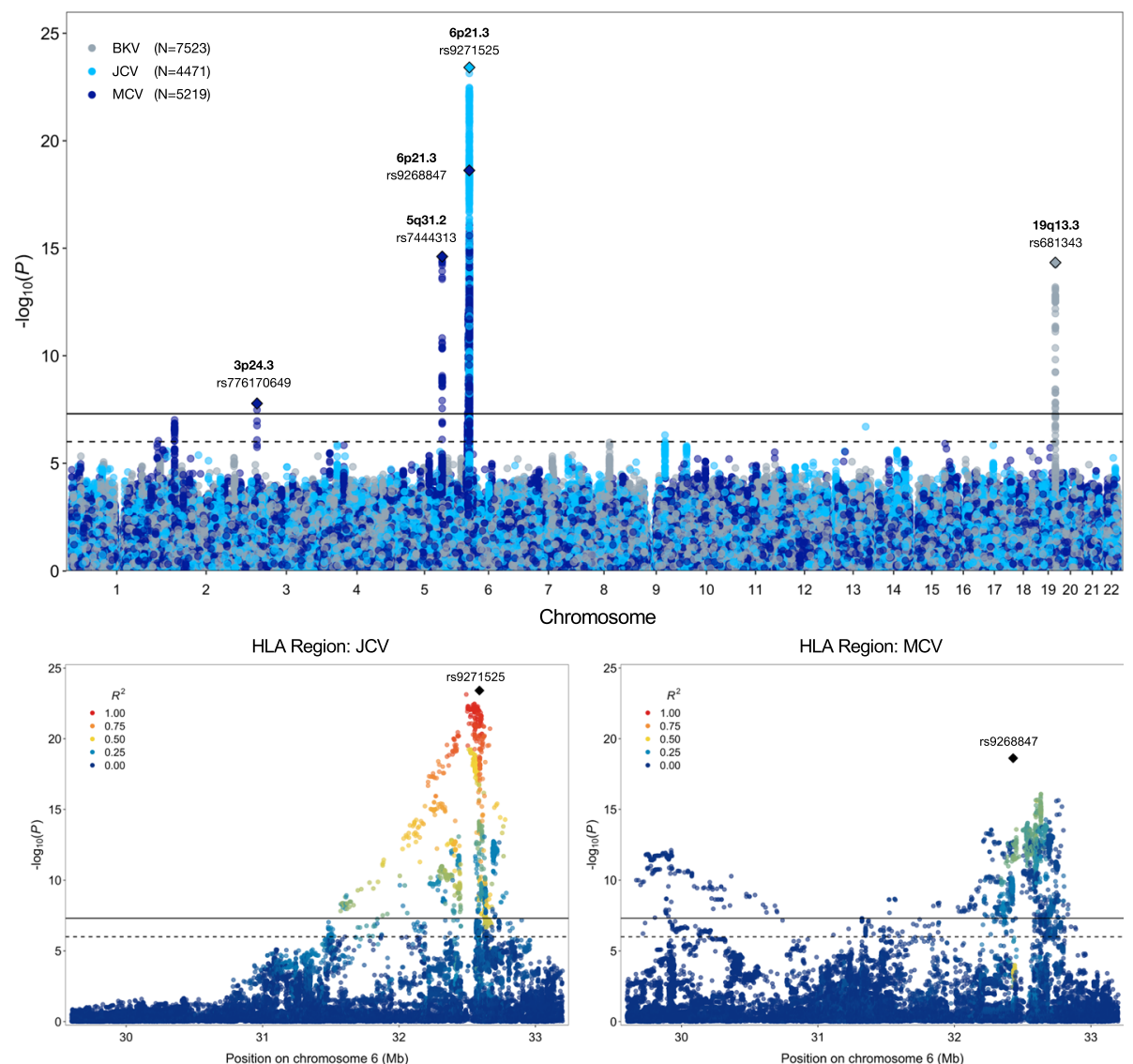

Fig. 2 Results from genome-wide and regional association analyses of continuous antibody response phenotypes (MFI z-scores) among individuals seropositive for human polyomaviruses BKV, JCV, and Merkel cell (MCV). The lower two panels depict the association signal and linkage disequilibrium (LD) structure in the HLA region for JCV and MCV

association with $H L A-D Q B 1$ expression in $C D 4+\mathrm{T}_{\mathrm{H}} 2$ cells was observed for rs9273325, 6:31486158_GT_G was an eQTL for ATP6V1G2 in naïve CD4+ $\mathrm{T}$ cells, and rs1130420 influenced the expression of 8 HLA class II genes in naïve $\mathrm{B}$ cells and $\mathrm{CD} 4+\mathrm{T}_{\mathrm{H}} 17$ cells.

We identified 7 significant $\left(p<5.0 \times 10^{-8}\right)$ protein quantitative trait loci (pQTL) for 38 proteins (Additional file 2: Table S4). Most of the pQTL targets were components of the adaptive immune response, such as the complement system (C4, CFB), chemokines (CCL15, CCL25), and defensin processing (Beta-defensin 19, Trypsin-3). The greatest number and diversity of pQTL targets $(n=16)$ was observed for rs681343, including BPIFB1, which plays a role in antimicrobial response in oral and nasal mucosa [45]; FUT3, which catalyzes the last step of Lewis antigen biosynthesis; and FGF19, part of the PI3K/Akt/MAPK signaling cascade that is dysregulated in cancer and neurodegenerative diseases [46].

\section{Cross-trait associations with disease outcomes}

To contextualize the relevance of genetic loci involved in infection response, we explored associations with selected cancers, schizophrenia, and that have a known or suspected viral etiology (Additional file 2: Table S5). The strongest secondary signal was observed for rs9273325 (HLA-DQB1), which was negatively associated with VZV antibody response and positively associated with schizophrenia susceptibility $\left(\mathrm{OR}=1.13, P=4.3 \times 10^{-15}\right)$. Other significant (Bonferroni $P<7.4 \times 10^{-4}$ ) associations with schizophrenia were detected for HSV1 (rs1130420: OR = 1.06, $P=1.8 \times 10^{-5}$ ), EBV EA-D (rs2647006: OR $=0.96$, $\left.P=2.7 \times 10^{-4}\right)$ JCV $\left(\right.$ rs9271525: OR $\left.=1.06, P=6.8 \times 10^{-5}\right)$, and BKV (rs681343: OR $=0.96, P=2.5 \times 10^{-4}$ ), with the latter being the only pleiotropic signal outside of HLA. Inverse associations with hematologic cancers were observed for HSV1 (rs1130420: $\left.\mathrm{OR}=0.89, P=3.5 \times 10^{-6}\right)$, VZV (rs9273325: $\mathrm{OR}=0.88, P=4.4 \times 10^{-5}$ ), and EBV EBNA (rs9269233: $\mathrm{OR}=0.88, P=2.7 \times 10^{-4}$ ) variants. HSV1 antibody response was also linked to Alzheimer's disease (rs1130420: $P=1.2 \times 10^{-4}$ ).

\section{Regional HLA associations}

Associations within the HLA region were refined by identifying independent (LD $r^{2}<0.05$ within $\pm 500 \mathrm{~kb}$ ) 
index variants with $P<5.0 \times 10^{-8}$ for each antigen response phenotype (Additional file 2: Table S6). Clumping seropositivity associations with respect to lead antibody response variants did not retain any loci, suggesting non-independence in signals for infection and reactivity for the same antigen. This was also confirmed based on genetic correlations $\left(r_{\mathrm{g}}\right)$ estimated using LD score regression [47], which ranged from $r_{\mathrm{g}}=0.407(p=$ $\left.5.1 \times 10^{-3}\right)$ for VZV to $r_{\mathrm{g}}=0.896\left(p=3.1 \times 10^{-7}\right)$ for $\mathrm{MCV}$. For this reason, all subsequent analyses focus on seroreactivity phenotypes. Clumping across phenotypes to assess the independence of HLA associations for different antigens identified 40 independent index variants: EBV EBNA (12), VZV (11), EBV ZEBRA (8), EBV p18 (5), MCV (3), and EBV EA-D (1) (Additional file 2: Table S7). No LD clumps were anchored by variants detected for CMV pp52, HHV7, HSV1, or JCV, suggesting that the HLA signals for these antigens are captured by lead loci for other phenotypes. The largest region with the lowest $p$ value was anchored by rs $9274728(P=4.7 \times$ $10^{-67}$ ) near $H L A-D Q B 1$, originally detected for EBV ZEBRA. Of the $11 \mathrm{VZV}$-associated variants, the largest clump was formed around rs4990036 $\left(P=4.5 \times 10^{-26}\right)$ in $H L A-B$.

Iterative conditional analyses adjusting for the HLA $\mathrm{SNP} /$ indel with the lowest $p$ value were performed until no variants remained with $P_{\text {cond }}<5.0 \times 10^{-8}$. Additional independent variants were identified for EBV EBNA (rs139299944, rs6457711, rs9273358, rs28414666, rs3097671), EBV ZEBRA (rs2904758, rs35683320, rs1383258), EBV p18 (rs6917363, rs9271325, rs66479476), and MCV (rs148584120, rs4148874) (Fig. 3; Additional file 2: Table S8). For CMV pp52, HHV7, HSV1, JCV, and VZV, the regional HLA signal was captured by the top GWAS variant (Fig. 2; Additional file 2: Table S8).

Next, we tested 101 classical HLA alleles and performed analogous iterative conditional analyses for significantly associated variants $\left(P<4.6 \times 10^{-5}\right)$. To help with the interpretation of our results, we depict the LD structure for HLA alleles in class II genes in Additional file 1: Figure S6. Significant associations across viruses were predominantly observed for class II HLA alleles. Five statistically independent signals were identified for antibody response to EBV ZEBRA (DRB4*00:00: $\beta=-$ $0.246, P=1.4 \times 10^{-46}$; DQB1*04:02: $\beta_{\text {cond }}=0.504, P_{\text {cond }}=$ $1.0 \times 10^{-19} ;$ DRB1*04:04: $\beta_{\text {cond }}=0.376, \quad P_{\text {cond }}=1.1 \times$ $10^{-18}$; DQA1*02:01: $\beta_{\text {cond }}=0.187, P_{\text {cond }}=1.1 \times 10^{-10}$; A*03:01: $\beta_{\text {cond }}=0.129, P_{\text {cond }}=1.9 \times 10^{-8}$ ) (Fig. 3 ; Additional file 2: Table S9). DRB4*00:00 represents copy number absence, which co-occurs with DRB1*04 and DRB1*07 alleles [48]. This is consistent with the magnitude and direction of unconditional associations observed for DRB1*07:01 $\left(\beta=0.251, P=1.3 \times 10^{-26}\right)$ and DRB4:04:01 $\quad\left(\beta=0.293, \quad P=7.9 \times 10^{-22}\right)$. Five conditionally independent alleles were also identified for EBV EBNA: DRB5*00:00: $\beta=-0.246, P=8.7 \times 10^{-30}$; DRB3*02:02: $\beta_{\text {cond }}=0.276, P_{\text {cond }}=6.8 \times 10^{-30}$; DQB1*02: $01: \beta_{\text {cond }}=-0.164, P_{\text {cond }}=3.6 \times 10^{-12} ;$ DRB4*00:00: $\beta=$ $0.176, P_{\text {cond }}=8.3 \times 10^{-17}$; and DPB1\%03:01: $\beta_{\text {cond }}=-$ $0.220, P_{\text {cond }}=4.7 \times 10^{-14}$ (Fig. 3 ; Additional file 2: Table S10). DRB5*00:00 denotes a copy number absence that sits on a common haplotype comprised of DRB1*15:01, DQB1*06:02, DQA1*01:02 [48], which may also include DRB5*01:01 [49] (Additional file 1: Figure S6). The presence of the DRB1*15:01-DQB1*06:02-DQA1*01:02 haplotype was associated with increased EBV EBNA seroreactivity $\left(\beta=0.330, P=2.5 \times 10^{-28}\right)$. Fewer independent alleles were observed for EBV p18 (DRB5*00: 00: $\beta=-0.210, P=1.7 \times 10^{-22}$; DRB1*04:04: $\beta_{\text {cond }}=$ $0.357, P_{\text {cond }}=1.3 \times 10^{-18}$ ) (Fig. 3; Additional file 2: Tables S11).

DQB1*02:01 was the only independently associated allele for EBV EA-D $\left(\beta=-0.154, P=8.4 \times 10^{-11}\right)$ and HSV1 $\left(\beta=0.145, P=2.8 \times 10^{-8}\right)$, although its effects were in opposite directions for each antigen (Additional file 2: Table S12). For VZV, associations with 16 classical alleles were accounted for by DRB1*03:01 $(\beta=0.236$, $\left.P=7.3 \times 10^{-26}\right)$. JCV shared the same lead allele as EBV EBNA and EBV p18 (DRB5*00:00: $\beta=0.350, P=1.2 \times$ $10^{-21}$ ) (Additional file 2: Table S12). Four conditionally independent signals were identified for MCV (DQA1*01: 01: $\beta=0.215, P=1.1 \times 10^{-15}$; DRB1*04:04: $\beta_{\text {cond }}=-$ $0.362, P_{\text {cond }}=3.0 \times 10^{-11} ; \mathrm{A}^{*} 29: 02: \beta_{\text {cond }}=-0.350, P=$ $1.0 \times 10^{-11}$; DRB1*15:01: $\beta_{\text {cond }}=-0.203, P=3.7 \times 10^{-12}$ ) (Fig. 3; Additional file 2: Table S13). Lastly, we integrated associations across variant types by including conditionally independent HLA alleles as covariates in the SNP-based analysis. With the exception of EBV antigens and HHV7, classical HLA alleles captured all genome-wide significant SNP signals (Additional file 1: Figure S7).

Finally, we tested 980 HLA amino acid substitutions (Additional file 2: Tables S14-S23), followed by omnibus haplotype tests at each position that had a significant amino acid and more than two possible alleles. The strongest allele-specific and haplotype associations were found at different positions in the same protein for EBV p18 (DR $\beta 1$ Ala -17 : $\beta=-0.194, P=1.0 \times 10^{-21}$; DR $\beta 1$ (13): $P_{\text {omni }}=4.6 \times 10^{-22}$; Additional file 2 : Table S14), MCV (DQ $\beta 1$ Leu-26: $\beta=-0.173, P=7.0 \times 10^{-18}$; DQ $\beta 1$ (125): $P_{\text {omni }}=2.0 \times 10^{-17}$; Additional file 2: Table S15), HHV7 (DQ $\beta 1$ His-30: $\beta=-0.111, P=1.2 \times 10^{-8}$; DQ $\beta 1$ (57): $P_{\text {omni }}=5.6 \times 10^{-9}$; Additional file 2 : Table S16), and HHV6 IE1B at (DR $\beta 1$ Ile-67: $\beta=0.131, P=1.6 \times 10^{-8}$; DR $\beta 1$ (13): $P_{\text {omni }}=1.1 \times 10^{-5}$; Additional file 2 : Table S17).

The strongest residue-specific and haplotype associations mapped to the same amino acid position for four 

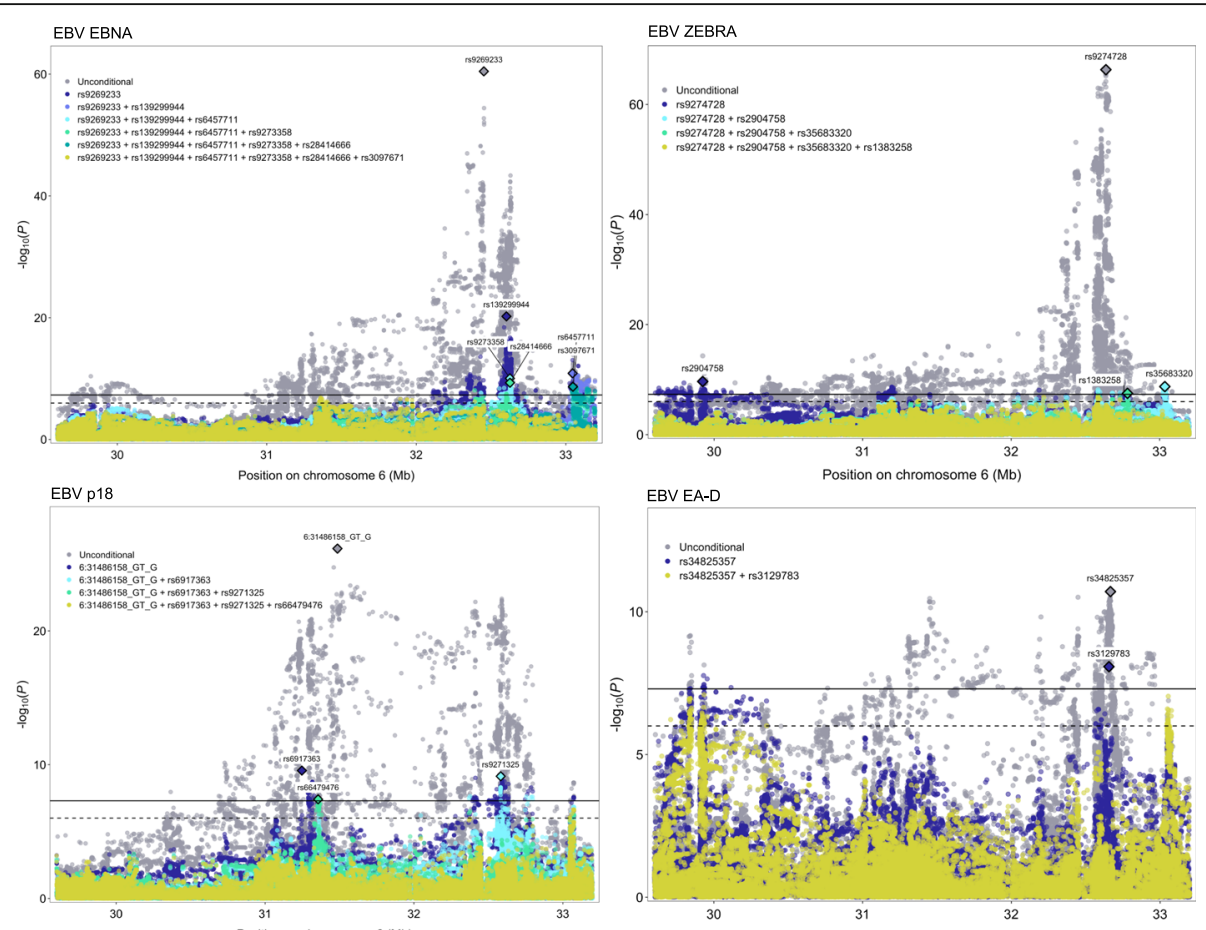

EBV EA-D
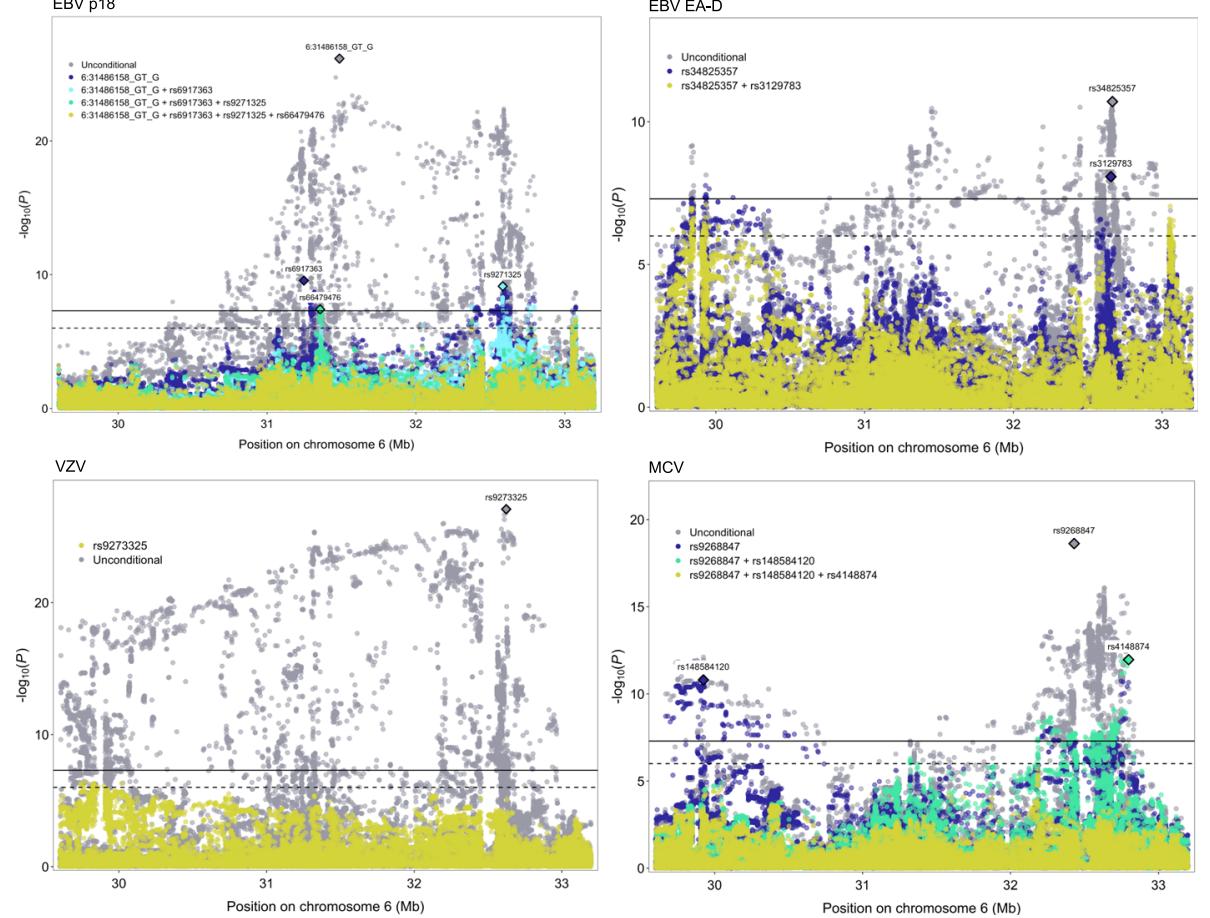

Fig. 3 Regional association plots for conditionally independent HLA genetic variants that were significantly $\left(P<5.0 \times 10^{-8}\right.$, solid black line) associated with each continuous antibody response phenotype. The suggestive significance threshold corresponds to $P<1.0 \times 10^{-6}$ (dotted black line)

phenotypes: EBV ZEBRA (Additional file 2: Table S18), HHV6 IE1A (Additional file 2: Table S17), HSV1 (Additional file 2: Table S19), and JCV (Additional file 2: Table S20). Amino acid residues at DQ $\alpha 1$ (175) were associated with antibody response to EBV ZEBRA (Glu: $\left.\beta=0.279, P=1.1 \times 10^{-61} ; P_{\text {omni }}=8.3 \times 10^{-62}\right)$. Glu-175 is present in DQA1*02:01 $\left(P=4.9 \times 10^{-27}\right)$, DQA1*03:01 $\left(P=1.3 \times 10^{-16}\right)$, and DQA1*04:01 $\left(P=1.9 \times 10^{-12}\right)$ and seems to better summarize the EBV ZEBRA signal at this locus. Substitutions in DR $\beta 1$ (96) contained the strongest predictors of JCV seroreactivity (His or Tyr: $\left.\beta=0.325, P=1.6 \times 10^{-25} ; P_{\text {omni }}=7.7 \times 10^{-23}\right)$. His- $96 /$ Tyr-96 are in high LD $\left(r^{2}=0.92\right)$ with DRB5*00:00, the top JCV-associated allele. However, this might mask the signal for Gln-96 $\left(\beta=-0.310, P=9.0 \times 10^{-23}\right)$, which is part of the DRB1*15:01 sequence $(\beta=-0.309, P=9.0 \times$
$10^{-21}$; LD $\left.r^{2}=0.94\right)$. The lead signal for HSV1 mapped to DQ $\beta 1$ (57) (Ala: $\beta=0.123, P=2.2 \times 10^{-10}$; $P_{\text {omni }}=$ $\left.6.5 \times 10^{-9}\right)$, which aligns with the association for the lead HSV1-allele DQB1*02:01.

For EBV EBNA, the strongest haplotype association was in DR $\beta 1(37)\left(P_{\text {omni }}=1.1 \times 10^{-55}\right)$, while the residue with the lowest $p$ value was DQ $\beta 1$ Ala-57 $(\beta=-0.237$, $P=1.4 \times 10^{-42}$ ) (Additional file 2: Table S21). Ala-57 maps to multiple DQB1 alleles and achieved a stronger signal for EBV EBNA than any classical HLA allele. Asp-9 in HLA-B showed the strongest association with antibody response to EBV EA-D $(\beta=-0.146, P=$ $1.8 \times 10^{-9}$; Additional file 2 : Table S22) and VZV $(\beta=$ 0.237, $P=9.7 \times 10^{-25}$; Additional file 2: Table S23). This amino acid sequence is part of $\mathrm{B} * 08: 01$, which had analogous effects on both phenotypes (EBV EA-D: 


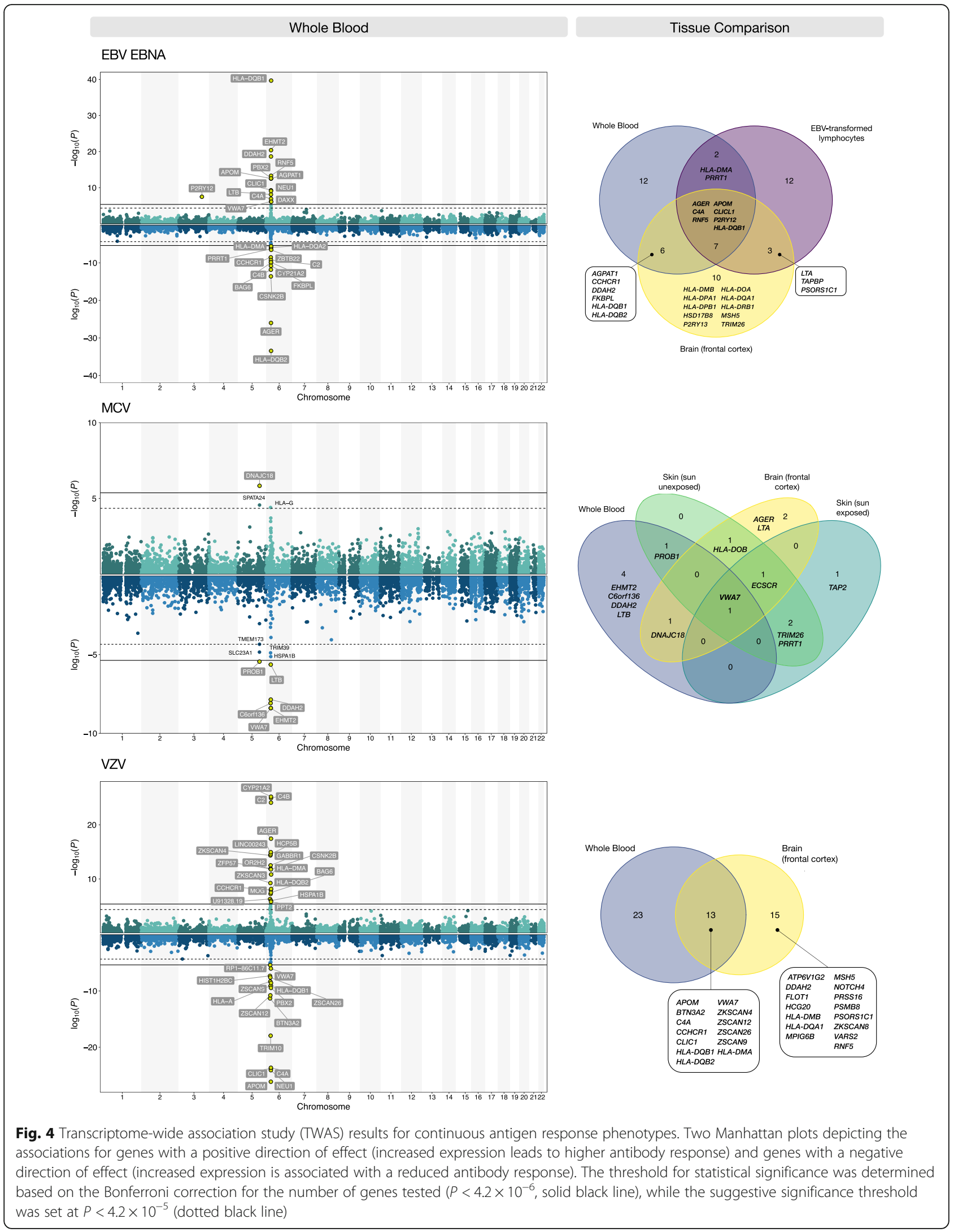


$\beta=-0.144, P=2.7 \times 10^{-9} ; \mathrm{VZV}: \beta=0.238, P=4.7 \times$ $10^{-25}$ ). Haplotypes with the lowest overall $p$ values were found in DQ $\beta 1(71)$ for $\operatorname{VZV~}\left(P_{\text {omni }}=9.8 \times 10^{-19}\right)$ and DR $\beta 1$ (11) for EBV EA-D $\left(P_{\text {omni }}=1.7 \times 10^{-10}\right)$.

\section{TWAS of genes involved in antibody response}

Based on known targets of infection or related pathologies, we considered expression in the frontal cortex (Additional file 2: Table S24), EBV-transformed lymphocytes for EBV antigens (Additional file 2: Table S25), and skin for MCV (Additional file 2: Table S26). Concordance across tissues was summarized using Venn diagrams (Fig. 4; Additional file 1: Figure S8). TWAS identified 114 genes significantly associated $\left(P_{\text {TWAS }}<\right.$ $4.2 \times 10^{-6}$ ) with antibody response in at least one tissue, 54 of which were associated with a single phenotype, while 60 influenced seroreactivity to multiple antigens. We also include results for 87 additional suggestively $\left(P_{\text {TWAS }}<4.2 \times 10^{-5}\right)$ associated genes.

The TWAS results included a predominance of associations in HLA class II genes. Some of the strongest overall associations were observed for HLA-DRB5 (EBV ZEBRA: $\left.P_{\text {cortex }}=4.2 \times 10^{-45}\right)$ and $H L A-D R B 1\left(\right.$ EBV EBNA: $P_{\text {cortex }}=$ $6.7 \times 10^{-39}$; EBV ZEBRA: $P_{\text {cortex }}=3.3 \times 10^{-33}$; JCV: $P_{\text {cortex }}=$ $6.5 \times 10^{-14}$; EBV p18: $P_{\text {cortex }}=2.2 \times 10^{-12}$ ). Increased expression of $H L A-D Q B 2$ was positively associated with antibody response to EBV ZEBRA $\left(P_{\text {blood }}=7.6 \times 10^{-19}\right)$, JCV $\left(P_{\text {blood }}=9.9 \times 10^{-10}\right), \operatorname{VZV~}\left(P_{\text {blood }}=7.0 \times 10^{-9}\right)$, HHV7 $\left(P_{\text {blood }}=7.3 \times 10^{-8}\right)$, and HSV1 $\left(P_{\text {blood }}=3.3 \times 10^{-7}\right)$, but negatively associated with EBV EBNA $\left(P_{\text {blood }}=3.6 \times 10^{-34}\right)$ and EBV p18 $\left(P_{\text {blood }}=2.1 \times 10^{-8}\right)$, in a consistent manner across tissues. The opposite was observed for $H L A-D Q B 1$,
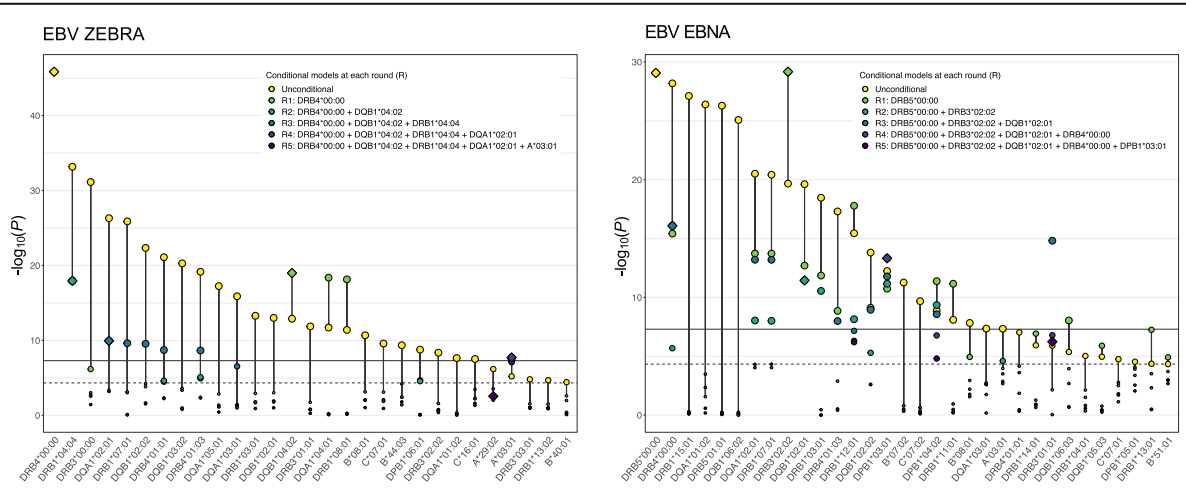

\begin{tabular}{ccccc} 
Allele & Frequency & Beta & $(\mathrm{SE})$ & $P_{\text {cond }}$ \\
\hline DRB4*00:00 & 0.652 & -0.246 & $(0.017)$ & $1.4 \times 10^{-46}$ \\
DQB1*04:02 & 0.020 & 0.504 & $(0.055)$ & $1.0 \times 10^{-19}$ \\
DRB1*04:04 & 0.041 & 0.376 & $(0.042)$ & $1.1 \times 10^{-18}$ \\
DQA1*02:01 & 0.145 & 0.187 & $(0.029)$ & $1.1 \times 10^{-10}$ \\
A $^{*} 03: 01$ & 0.144 & 0.129 & $(0.023)$ & $1.9 \times 10^{-8}$
\end{tabular}

\begin{tabular}{ccrcc} 
Allele & Frequency & Beta & $(\mathrm{SE})$ & $P_{\text {cond }}$ \\
\hline DRB5 $^{*} 00: 00$ & 0.845 & -0.255 & $(0.022)$ & $8.7 \times 10^{-30}$ \\
DRB3 $^{*} 02: 02$ & 0.136 & 0.276 & $(0.024)$ & $6.8 \times 10^{-30}$ \\
DQB1 $^{*} 02: 01$ & 0.149 & -0.164 & $(0.023)$ & $3.6 \times 10^{-12}$ \\
DRB4 $^{*} 99: 01$ & 0.652 & 0.176 & $(0.021)$ & $8.3 \times 10^{-17}$ \\
DPB1 $^{*} 03: 01$ & 0.100 & -0.220 & $(0.029)$ & $4.7 \times 10^{-14}$
\end{tabular}
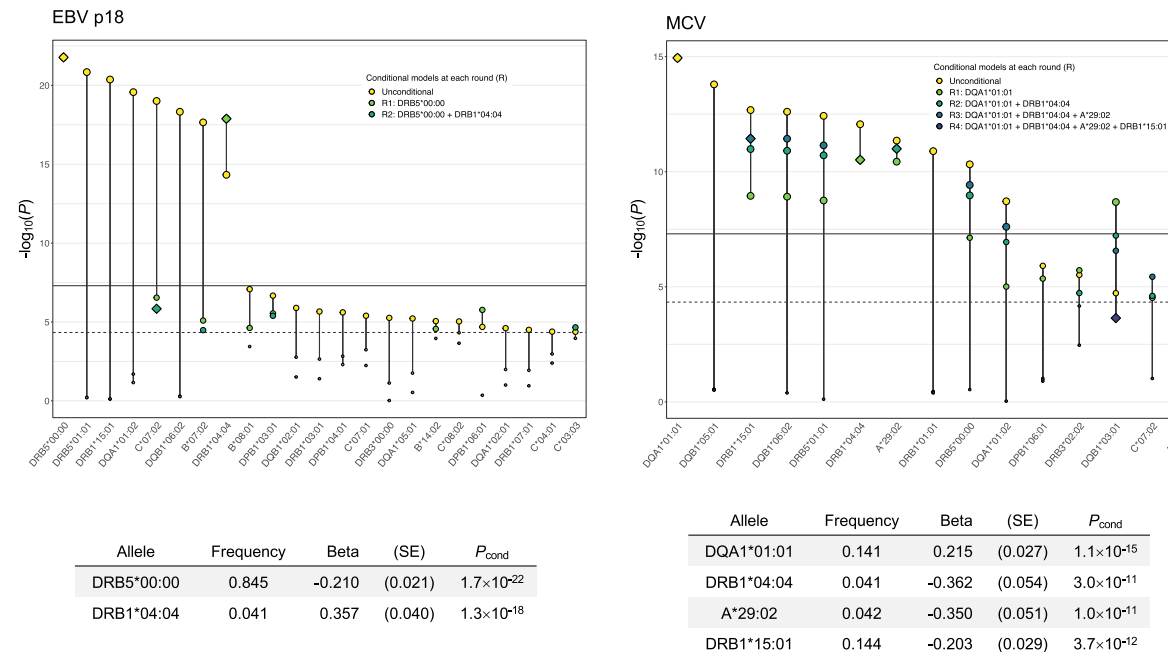

Fig. 5 Conditionally independent classical HLA alleles significantly $\left(P_{\text {cond }}<5.0 \times 10^{-8}\right.$, solid line) associated with each continuous antibody response phenotype. Only classical alleles that surpassed the Bonferroni-corrected significance threshold $\left(P<4.6 \times 10^{-5}\right.$, dotted line) were included in conditional analyses 
with positive effects on EBV EBNA and EBV p18 and inverse associations with EBV ZEBRA, JCV, VZV, HHV7, and HSV1.

The TWAS analyses also identified a number of significant associations in the HLA class III region that were not detected in other analyses. The top-ranking VZV associated gene was APOM $\left(P_{\text {blood }}=7.5 \times 10^{-27}\right.$, $\left.P_{\text {cortex }}=1.1 \times 10^{-25}\right)$. Interestingly, opposite directions of effect were observed for $C 4 A$ and $C 4 B$ gene expression. Increased $C 4 A$ expression was positively associated with all EBV antigens (Additional file 2: Table S25), but negatively associated with $\operatorname{VZV~}\left(P_{\text {blood }}=2.3 \times 10^{-24}\right)$ and HSV1 $\left(P_{\text {cortex }}=1.8 \times 10^{-5}\right)$ antibody levels (Additional file 2: Table S24). On the other hand, increased $C 4 B$ expression was inversely associated with EBV phenotypes, but positively associated with VZV $\left(P_{\text {blood }}=8.1 \times 10^{-25}\right)$ and HSV1 $\left(P_{\text {blood }}=1.1 \times 10^{-5}\right)$. A similar pattern was also observed for CYP21A2 and $C 2$, with positive effects on antibody response to VZV and HSV1, and negative effects for all EBV antigens. Other novel TWAS findings were detected for HHV7 in 22q13.2 (CTA-223H9.9: $P_{\text {TWAS }}=2.5 \times 10^{-6} ; C S D C 2: P_{\text {TWAS }}=3.0 \times 10^{-6} ;$ TEF $:$ $\left.P_{\text {TWAS }}=3.1 \times 10^{-6}\right)$ and $1 \mathrm{q} 31.2\left(\right.$ RGS1: $P_{\text {TWAS }}=3.3 \times$ $\left.10^{-6}\right)$.

The TWAS recapitulated several GWAS-identified loci: 3q25.1 for EBV EBNA ( $P 2 R Y 13$ : $P_{\text {cortex }}=1.1 \times 10^{-8}$; P2RY12: $P_{\text {blood }}=3.3 \times 10^{-8}$ ) and $19 \mathrm{q} 13.33$ for BKV (FUT2: $P_{\text {TWAS }}=8.1 \times 10^{-13} ;$ NTN5: $\left.P_{\text {TWAS }}=1.1 \times 10^{-9}\right)$. Transcriptomic profiles in skin tissues provided supporting evidence for the role of multiple genes in $5 \mathrm{q} 31.2$ in modulating MCV antibody response (Fig. 5; Additional file 2: Table S26). The strongest signal was observed in for $E C S C R$ (skin sun unexposed: $P_{\text {TWAS }}=5.0 \times 10^{-15}$; skin sun exposed: $P_{\text {TWAS }}=4.2 \times 10^{-13}$ ), followed by PROB1 (sun unexposed: $P_{\text {TWAS }}=1.5 \times 10^{-11}$ ). ECSCR expression was also associated based on expression in the frontal cortex, while PROB1 exhibited a significant, but attenuated effect in whole blood. VWA7 was the only gene associated across all four tissues for MCV and was also associated with antibody response to several EBV antigens.

Comparison of results for seroreactivity and seropositivity revealed a number of genes implicated in both steps of the infection process (Additional file 2: Table S27). Associations with HLA DQA and DQB genes in whole blood and HLA-DRB genes in the frontal cortex were observed for EBV antigens, JCV, and MCV. For $\mathrm{MCV}$, the strongest seropositivity signals were observed for HLA class III genes AGER $\left(P_{\text {cortex }}=9.0 \times 10^{-21}\right)$ and EHMT2 $\left(P_{\text {blood }}=5.8 \times 10^{-18}\right)$, which were also among the top-ranking genes for seroreactivity. Increased ECSC $R$ expression conferred an increased susceptibility to MCV infection $\left(P_{\text {cortex }}=1.8 \times 10^{-8}\right)$, mirroring its effect on seroreactivity. In contrast to antibody response, no significant associations with any HLA genes were observed for VZV seropositivity.

Analyses using the Reactome database identified significant $\left(q_{\mathrm{FDR}}<0.05\right)$ enrichment for TWAS-identified genes in pathways involved in initiating antiviral responses, such as MHC class II antigen presentation, TCR signaling, and interferon (IFN) signaling (Additional file 1: Figure S9). Pathways unique to herpesviruses included folding, assembly, and peptide loading of class I MHC $\left(q=3.2 \times 10^{-7}\right)$ and initial triggering of complement $\left(q=9.8 \times 10^{-3}\right)$. Polyomaviruses were associated with the non-canonical nuclear factor (NF)- $\mathrm{kB}$ pathway activated by tumor necrosis factor (TNF) superfamily $\left(q=1.9 \times 10^{-3}\right)$.

\section{Discussion}

We performed genome-wide and transcriptome-wide association studies for serological phenotypes for 16 common viruses in a well-characterized, populationbased cohort. We discovered novel genetic determinants of viral antibody response beyond the HLA region for BKV, MCV, HHV7, and EBV EBNA. Consistent with previous studies $[7,8]$, we detected strong signals for immune response to diverse viral antigens in the HLA region, with a predominance of associations observed for alleles and amino acids in $H L A-D R B 1$ and $H L A-D Q B 1$, as well as transcriptome-level associations for multiple class II and III HLA genes. Taken together, the findings of this work provide a resource for further understanding the complex interplay between viruses and the human genome, as well as a first step towards understanding genetic determinants of reactivity to common infections.

One of our main findings is the discovery of $5 \mathrm{q} 31.2$ as a susceptibility locus for MCV infection and MCV antibody response, implicating two main genes: TMEM173 (or STIN G1) and ECSCR. The former encodes STING (stimulator of interferon genes), an endoplasmic reticulum (ER) protein that controls the transcription of host defense genes and plays a critical role in response to DNA and RNA viruses [50]. STING is activated by cyclic GMP-AMP synthase (cGAS), a cytosolic DNA sensor that mounts a response to invading pathogens by inducing IFN1 and NF- $\mathrm{kB}$ signaling $[51,52]$. Polyomaviruses penetrate the ER membrane during cell entry, a process that may be unique to this viral family [53], which may trigger STING signaling in a distinct manner from other viruses [53]. Multiple cancer-causing viruses, such as KSHV, HBV, and HPV18, encode oncoproteins that disrupt cGAS-STING activity, which illustrates the evolutionary pressure on DNA tumor viruses to develop functions against this pathway and its importance in carcinogenesis [51]. Furthermore, cGAS-STING activation has been shown to trigger antitumor $\mathrm{T}$ cell responses, a mechanism that can be leveraged by targeted immunotherapies [54-56]. Several studies suggest STING agonists may 
be effective against tumors resistant to PD-1 blockade, as well as promising adjuvants in cancer vaccines [57-59].

ECSCR expression in skin and brain tissues was associated with MCV antibody response and infection. This gene encodes an endothelial cell-specific chemotaxis regulator, which plays a role in angiogenesis and apoptosis [60]. ECSCR is a negative regulator of PI3K/Akt signaling by enhancing membrane localization of PTEN and operates in tandem with VEGFR-2 and other receptor tyrosine kinases [61]. In addition to 5q31.2, another novel MCV seroreactivity associated region was identified in 3p24.3, anchored by rs776170649, which has been linked to platelet phenotypes [62]. These findings align with a role of platelet activation in defense against infections via degranulation-mediated release of chemokines and $\beta$-defensin [63].

Genetic variation within Fucosyltransferase 2 (FUT2) has been studied extensively in the context of human infections; however, its effect on BKV seroreactivity is novel. Homozygotes for the nonsense mutation (rs601338 G>A) that inactivates the FUT2 enzyme are unable to secrete $\mathrm{ABO}(\mathrm{H})$ histo-blood group antigens or express them on mucosal surfaces $[64,65]$. The allele which confers increased BKV antibody response (rs681343-T) is in LD $\left(r^{2}=1.00\right)$ with rs601338-A, the non-secretor allele, which confers resistance to norovirus [66, 67], rotavirus [68], Helicobacter pylori [69], childhood ear infection, mumps, and common colds [13]. However, increased susceptibility to other pathogens, such as meningococcus and pneumococcus [70], has also been observed in non-secretors. Isolating the underlying mechanisms for BKV response is challenging because FUT2 is a pleiotropic locus associated with diverse phenotypes, including autoimmune and inflammatory conditions [71, 72], serum lipids [73], B vitamins [65, 74], alcohol consumption [75], and even certain cancers [76]. In addition to FUT2 in 19q13.33, NTN5 (netrin 5) suggests a possible link between BKV and neurological conditions. NTN5 is primarily expressed in neuroproliferative areas, suggesting a role in adult neurogenesis, which is dysregulated in glioblastoma and Alzheimer's disease $[77,78]$.

We also report the first GWAS of serological phenotypes for HHV7. Genetic determinants of HHV7 antibody response in $6 \mathrm{p} 21.32$ were predominantly localized in $H L A-D Q A 1$ and $H L A-D Q B 1$, with associations similar to other herpesviruses. In 11q23.3, rs75438046 maps to the 3' UTR of CXCR5, which controls viral infection in B cell follicles [79], and $B C L 9 L$, a translocation target in acute lymphoblastic leukemia [80] and transcriptional activator of the $\mathrm{Wnt} / \beta$-catenin cancer signaling pathway [81]. In 17q21.32, TBKBP1 encodes an adaptor protein that binds to TBK1 and is part of the TNF/NF- $\mathrm{KB}$ interaction network, where it regulates immune responses to infectious triggers, such as IFN1 signaling [82].
Interestingly, a protein interactome map recently revealed that SARS-CoV-2 nonstructural protein 13 (Nsp13) includes TBK1-TBKBP1 among its targets [83]. Other functions of the TBK1-TBKBP1 axis relate to tumor growth and immunosuppression through induction of PD-L1 [84].

Several additional genes involved in HHV7 immune response were identified in TWAS. TEF in 22q13.2 is an apoptotic regulator of hematopoietic progenitors with tumor promoting effects mediated by inhibition of G1/S cell cycle transition and Akt/FOXO signaling [85]. RGS1 in 1q31.2 has been linked to multiple autoimmune diseases, including multiple sclerosis [86], as well as poor prognosis in melanoma and diffuse large B cell lymphoma mediated by inactivation of Akt/ERK $[87,88]$.

Other genes outside of the HLA region associated with viral infection response were detected for EBV EBNA in 3q25.1. The lead variant (rs67886110) is an eQTL for MED12L and P2RY12 genes, which have been linked to neurodegenerative conditions [89, 90]. P2RY12 and $P 2 R Y 13$, identified in TWAS, are purinergic receptor genes that regulate microglia homeostasis and have been implicated in Alzheimer's susceptibility via inflammatory and neurotrophic mechanisms [90].

Considering genetic variation within the HLA region, our results confirm its pivotal role at the interface of host pathogen interactions and highlight the extensive sharing of HLA variants that mediate these interactions across virus families and antigens. Genes in this region code for cell-surface proteins that facilitate antigenic peptide presentation to immune cells that regulate responses to invading pathogens. This region is critical for adaptive immune response but also has significant overlap with susceptibility alleles for autoimmune diseases. We identified 40 independent SNPs/indels associated with EBV (EBNA, EA-D, VCA p18, and ZEBRA), VZV, and $M C V$ antibody response that accounted for all significant HLA associations for other phenotypes. However, compared to conditional analyses, clumping may overestimate the number of independent variants due to the complex long-range LD structure in HLA and violation of the assumption that $500-\mathrm{kb}$ windows are sufficient to exclude correlated variants in this region. Of the 14 conditionally independent, genome-wide significant classical alleles identified for 10 antigens, 7 were associated with multiple phenotypes. The most commonly shared HLA alleles were DRB5*00:00, DRB1*04:04, an known rheumatoid arthritis risk allele [91], and DQB1*02:01, associated with celiac disease risk [92]. Although allele absence represented by DRB5*00:00 may have a functional role in altering antigen response, a more likely explanation for the observed signal is that DRB5*00:00 acts as a biomarker for a specific class II haplotype. Rather than having a direct causal effect on 
antigen presentation, this "null allele" summarizes signals from multiple HLA loci, including the extended DRB5*01:01-DRB1*15:01-DQB1*06:02-DQA1*01:02 haplotype that has been implicated in the etiology of multiple autoimmune diseases and EBV EBNA IgG levels. DRB1*15:01-DQB1*06:02-DQA1*01:02 is protective for type 1 diabetes [93], while DRB5*01:01-DRB15:01 confers the strongest risk for developing multiple sclerosis [86]. Amino acid residues in DR $\beta 1$ at positions 11, 13, 71, and 74 and in DQ $\beta 1$ codon 57 represent established susceptibility loci for rheumatoid arthritis [94], type 1 diabetes [95], and multiple sclerosis [96] that exhibited strong associations with IgG levels for EBV, HHV7, VZV, JCV, and MCV antigens, and in some cases harbored the top signal of all HLA variants. Further research is needed to delineate shared genetic pathways that invoke autoimmunity and influence viral response.

Despite the predominance of association in HLA class II, several notable associations in HLA class I were detected. A*29:02 conferred reduced MCV seroreactivity and its sequence overlaps with amino acid residues in the A $\alpha 1$ domain (Thr-9, Leu-62, Gln-63, Asn-77, and Met-97) that were also significantly associated with decreased MCV antibody response. This is consistent with downregulation of MHC I as a potential mechanism through which Merkel cell tumors evade immune surveillance [97]. The strongest residue-specific signal for EBV EA-D and VZV mapped to B-Asp-9, which is located in the peptide binding groove and tags the $B * 08: 01$ allele, part of the HLA 8.1 ancestral haplotype. There is extensive evidence linking HLA 8.1, and B*08:01 specifically, with autoimmune diseases [98] and certain cancers $[99,100]$, which may be attributed to its high cell-surface stability and increased probability of CD8+ $\mathrm{T}$ cell activation.

Comparison with other studies of host genetics and viral infection susceptibility shows that our results align with previously reported findings $[7-9,101]$ (Additional file 2: Table S28). We replicated most associations from two of the largest GWAS of humoral immune response in European ancestry subjects by Hammer et al. [7] $(n=$ 2363) and Scepanovic et al. [8] $(n=1000)$, including HLA SNPs, alleles, amino acids, and haplotypes linked to EBV EBNA IgG, MCV IgG and serostatus, and JCV serostatus. We also replicated two HLA-DRB1 variants (rs477515, rs2854275) associated with EBV EBNA antibody levels in a Mexican American population [9]. GWAS of HPV16 L1 replicated a variant previously linked to HPV8 seropositivity (rs9357152, $P=0.008,6$ ). Some of our findings contrast with Tian et al. [13], although we confirmed selected associations, such as A*02:01 (shingles) with VZV $\left(P=4.1 \times 10^{-8}\right)$ and rs2596465 (mononucleosis) with EBV EBNA $(P=3.3 \times$ $\left.10^{-9}\right)$ and EBV p18 $\left(P=1.0 \times 10^{-12}\right)$. These differences may be partly accounted for by self-reported disease status in Tian et al. which is likely to reflect symptom severity and may be an imprecise indicator of infection with certain viruses or the magnitude of antibody response to infection.

One of the most striking findings in SNP-based HLA analyses was the genome-wide significant association between rs9273325, index VZV antibody response variant, and risk of schizophrenia. Previous epidemiologic and serologic studies have linked infections to schizophrenia, although the underlying mechanisms remain to be elucidated [102]. Viruses are plausible etiologic candidates for schizophrenia due to their ability to invade the central nervous system and disrupt neurodevelopmental processes by targeting specific neurons, as well as the potential for latent infection to negatively impact plasticity and neurogenesis via pro-inflammatory and aberrant immune signaling [102, 103]. These observations are consistent with the established role the HLA region, including $H L A-D Q B 1$, in schizophrenia etiology [104, $105]$, and is further supported by previously reported associations for rs9273325 with blood cell traits [62] and immunoglobulin A deficiency [106], as well as its role as an eQTL for $H L A-D Q B 1$ in CD4+ $\mathrm{T}_{2} \mathrm{~h}$ cells. Schizophrenia susceptibility alleles DRB1*03:01 [104], DQB1*02:01, and B*08:01 were also the top three alleles associated with VZV antibody response in the unconditional analysis. Enhanced complement activity has been proposed as the mechanism mediating the synaptic loss and excessive pruning which is a hallmark of schizophrenia pathophysiology [107]. Complement component 4 (C4) alleles were found to increase risk of schizophrenia proportionally to their effect on increasing C4A expression in brain tissue [107]. Using gene expression models in whole blood and the frontal cortex we demonstrated that increased C4A expression is negatively associated with VZV antibody response. We also observed associations with $C 4 A$ and $C 4 B$ in EBV and HSV-1, but not other viruses. Taken together, these findings delineate a potential mechanism through which aberrant immune response to VZV infection, and potentially HSV-1 and EBV, may increase susceptibility to schizophrenia. However, cautious interpretation is warranted due to significant pleiotropy between HLA loci associated with viral infection and broad immune function.

Several limitations of this work should be noted. First, the UK Biobank is unrepresentative of the general UK population due to low participation resulting in healthy volunteer bias [108]. However, since the observed pattern of seroprevalence is consistent with previously published estimates [15], we believe the impact of this bias is likely to be minimal on genetic associations with serological phenotypes. Second, our analyses were 
restricted to participants of European ancestry due to limited serology data for other ancestries, which limits the generalizability of our findings to diverse populations. Third, we were unable to conduct formal statistical replication of novel GWAS and TWAS signals in an independent sample due to the lack of such a population. Nevertheless, our successful replication of multiple previously reported variants and, combined with the observation that newly discovered genes and variants are part of essential adaptive and innate immunity pathways, support the credibility of our findings. Lastly, we also stress caution in the interpretation of GWAS results for non-ubiquitous pathogens, such as $\mathrm{HBV}, \mathrm{HCV}$, and $\mathrm{HPV}$, due to a lack of information on exposure, as well as low numbers of seropositive individuals.

Our study also has distinct advantages. The large sample size of the UK Biobank facilitated more powerful genetic association analyses than previous studies, particularly in a population-based cohort unselected for disease status. Our detailed HLA analysis shows independent effects of specific HLA alleles and pleiotropic effects across multiple viruses. Analyses of genetic associations in external datasets further demonstrate a connection between host genetic factors influencing immune response to infection and susceptibility to cancers and neurological conditions.

The results of this work highlight widespread genetic pleiotropy between pathways involved in regulating humoral immune response to novel and common viruses, as well as complex diseases. The complex evolutionary relationship between viruses and humans is not dictated simply by infection and acute sickness, it is a complex nuanced architecture of initial challenge tempered with tolerance of viral latency over time. Yet it is that architecture that is evolutionarily optimized to maximize fitness early in life, the result of which may be increased risk for complex diseases later in life. Understanding this complex interplay through both targeted association studies and functional investigations between host genetic factors and immune response has implications for complex disease etiology and may facilitate the discovery of novel therapeutics in a wide range of diseases.

\section{Conclusions}

We present a genome-wide investigation host genetic factors influencing antibody response to common viral antigens. Our study confirms the importance of HLA class II genes in modulating IgG levels for human herpes and polyomaviruses and illustrates the complexity of genetic effects in this region represented by single variants, classical alleles, and amino acid substitutions. We also uncovered novel genetic loci beyond HLA that contribute to host-virus interaction, including signals in 3q25.1, 5q31.2, and 19q13.33, which may operate via direct effects on gene expression. Taken together, the findings presented here provide a resource of genetic determinants of immune response to common viruses, which may be leveraged in future studies of complex disease etiology and personalized therapeutics or vaccines.

\section{Supplementary information}

Supplementary information accompanies this paper at https://doi.org/10. 1186/s13073-020-00790-x.

Additional file 1. This folder contains the pdf file with all

Supplementary Figures (Figures S1-S9) that are referenced throughout the manuscript, with corresponding figure legends.

Additional file 2. This folder contains the pdf file with all Supplementary Tables (Tables S1-S28) referenced throughout the manuscript, with corresponding descriptions.

\section{Abbreviations}

BKV: BK polyomavirus; EBNA: Epstein-Barr nuclear antigen; EBV: Epstein-Barr virus; eQTL: Expression quantitative trait locus; GWAS: Genome-wide association study; HBV: Hepatitis B virus; HCV: Hepatitis C virus; HHV6: Human herpesvirus 6; HHV7: Human herpesvirus 7; HLA: Human leukocyte antigen; HPV16: Human papillomavirus 16; HPV18: Human papillomavirus 18; HSV1: Herpes simplex virus 1; HSV2: Herpes simplex virus; IgG: Immunoglobulin G; JCV: JC polyomavirus; MCV: Merkel cell polyomavirus; MFI: Median fluorescence intensity; OR: Odds ratio; SNP: Single nucleotide polymorphism; SQTL: Splicing quantitative trait locus; TWAS: Transcriptome-wide association study; UKB: UK Biobank; VZV: Varicella zoster virus

\section{Acknowledgements}

Not applicable.

\section{Authors' contributions}

Study conception: L.K., M.M., J.S.W.; development of analytic strategy: L.K., S.S.F, J.S.W.; genetic data processing and quality control: S.R.R., G.A.W.; imputation of gene expression profiles and HLA amino acid residues: L.K., T.B.C., Y.B., G.A.W.; statistical analysis: L.K.; manuscript was drafted by L.K. and S.S.F. with input from J.S.W. and E.Z. All authors contributed to the interpretation of the results and provided critical feedback on the manuscript. All authors read and approved the final manuscript.

\section{Funding}

This research was supported by funding from the National Institutes of Health (US NCI R25T CA112355 and R01 CA201358; PI: Witte). Maike Morrison was funded by the University of California San Francisco's Amgen Scholars Program.

\section{Availability of data and materials}

The data used in this study are available via a direct application to the UK Biobank (http://www.ukbiobank.ac.uk/register-apply/). Individual-level data are open access and can be obtained after completing a registration process and obtaining approval from the UK Biobank Data Access committee. Type 1 Diabetes Genetics Consortium (T1DGC) reference panel used for imputation with SNP2HLA was obtained via approved access from the National Institute of Diabetes and Digestive and Kidney Disease (NIDDK) Central Repository: https://repository.niddk.nih.gov/studies/t1dgc-special/. Gene expression prediction models used in transcriptome-wide association analyses are available from the PredictDB repository, published on Zenodo [37]: https://zenodo.org/record/3518299.

\section{Ethics approval and consent to participate}

This research was conducted with approved access to UK Biobank data under application number 14105 (PI: Witte). All UK Biobank participants provided informed consent at recruitment. The UK Biobank has been developed as a research resource that relies on a broad consent model. Research using UK Biobank data is conducted in accordance with the UK 
Biobank Ethics and Governance Framework, which sets specific standards for this resource so that all necessary safeguards are in place to ensure that the data and samples are only used for scientifically and ethically approved research. Ethics approval for the UK Biobank study was obtained from the North West Centre for Research Ethics Committee (11/NW/0382).

\section{Consent for publication}

Not applicable.

\section{Competing interests}

J.S.W. is a non-employee co-founder of Avail.bio and serves as an expert witness for Pfizer and Sanofi. The remaining authors declare that they have no competing interests.

\section{Author details}

1Department of Epidemiology and Biostatistics, University of California San Francisco, San Francisco, CA, USA. ${ }^{2}$ Department of Neurological Surgery, University of California San Francisco, San Francisco, CA, USA. ${ }^{3}$ Helen Diller Family Comprehensive Cancer Center, University of California, San Francisco, San Francisco, CA, USA. "Weill Institute for Neurosciences, University of California San Francisco, San Francisco, CA, USA. ${ }^{5}$ Department of Biology, Stanford University, Stanford, CA, USA. ${ }^{6}$ Summer Research Training Program, Graduate Division, University of California San Francisco, San Francisco, CA, USA. ${ }^{7}$ Department of Mathematics, The University of Texas, Austin, TX, USA. ${ }^{8}$ Department of Molecular Medicine, Université Laval, Institut Universitaire de Cardiologie et de Pneumologie de Québec, Quebec City, QC, Canada. ${ }^{9}$ Program in Biological and Medical Informatics, University of California San Francisco, San Francisco, CA, USA. ${ }^{10}$ Center for Applied Bioinformatics, St. Jude Children's Research Hospital, Memphis, TN, USA. " 1 Department of Medicine, University of California, San Francisco, San Francisco, CA, USA. ${ }^{12}$ Institute for Human Genetics, University of California San Francisco, San Francisco, CA, USA. ${ }^{13}$ Department of Urology, University of California San Francisco, San Francisco, CA, USA.

Received: 29 May 2020 Accepted: 7 October 2020

\section{Published online: 27 October 2020}

\section{References}

1. Aiewsakun $P$, Katzourakis A. Marine origin of retroviruses in the early Palaeozoic Era. Nat Commun. 2017;8:13954.

2. Wang W, Xu Y, Gao R, Lu R, Han K, Wu G, et al. Detection of SARS-CoV-2 in different types of clinical specimens. JAMA. 2020;323(18):1843-4. https://doi. org/10.1001/jama.2020.3786.

3. Moore PS, Chang Y. Why do viruses cause cancer? Highlights of the first century of human tumour virology. Nat Rev Cancer. 2010;10(12):878-89.

4. Engdahl E, Gustafsson R, Huang J, Bistrom M, Lima Bomfim I, Stridh P, et al. Increased serological response against human herpesvirus $6 \mathrm{~A}$ is associated with risk for multiple sclerosis. Front Immunol. 2019;10:2715.

5. Readhead B, Haure-Mirande JV, Funk CC, Richards MA, Shannon P, Haroutunian $V$, et al. Multiscale analysis of independent Alzheimer's cohorts finds disruption of molecular, genetic, and clinical networks by human herpesvirus. Neuron. 2018;99(1):64-82 e7.

6. Chen D, McKay JD, Clifford G, Gaborieau V, Chabrier A, Waterboer T, et al. Genome-wide association study of HPV seropositivity. Hum Mol Genet. 2011;20(23):4714-23.

7. Hammer C, Begemann M, McLaren PJ, Bartha I, Michel A, Klose B, et al. Amino acid variation in HLA class II proteins is a major determinant of humoral response to common viruses. Am J Hum Genet. 2015;97(5):738-43.

8. Scepanovic P, Alanio C, Hammer C, Hodel F, Bergstedt J, Patin E, et al. Human genetic variants and age are the strongest predictors of humoral immune responses to common pathogens and vaccines. Genome Med. 2018;10(1):59.

9. Rubicz R, Yolken R, Drigalenko E, Carless MA, Dyer TD, Bauman L, et al. A genome-wide integrative genomic study localizes genetic factors influencing antibodies against Epstein-Barr virus nuclear antigen 1 (EBNA-1). PLoS Genet. 2013;9(1):e1003147.

10. Liu S, Huang S, Chen F, Zhao L, Yuan Y, Francis SS, et al. Genomic analyses from non-invasive prenatal testing reveal genetic associations, patterns of viral infections, and Chinese population history. Cell. 2018;175(2):347-59 e14.

11. Besson C, Amiel C, Le-Pendeven C, Plancoulaine S, Bonnardel C, Ranque B, et al. Strong correlations of anti-viral capsid antigen antibody levels in first- degree relatives from families with Epstein-Barr virus-related lymphomas. J Infect Dis. 2009;199(8):1121-7.

12. Kenney AD, Dowdle JA, Bozzacco L, McMichael TM, St Gelais C, Panfil AR, et al. Human genetic determinants of viral diseases. Annu Rev Genet. 2017;51:241-63.

13. Tian C, Hromatka BS, Kiefer AK, Eriksson N, Noble SM, Tung JY, et al. Genomewide association and HLA region fine-mapping studies identify susceptibility loci for multiple common infections. Nat Commun. 2017;8(1):599.

14. Bycroft C, Freeman C, Petkova D, Band G, Elliott LT, Sharp K, et al. The UK Biobank resource with deep phenotyping and genomic data. Nature. 2018; 562(7726):203-9.

15. Mentzer AJ, Brenner N, Allen N, Littlejohns TJ, Chong AY, Cortes A, et al. Identification of host-pathogen-disease relationships using a scalable Multiplex Serology platform in UK Biobank. medRxiv. 2019:19004960. https:// doi.org/10.1101/19004960.

16. Manichaikul A, Mychaleckyj JC, Rich SS, Daly K, Sale M, Chen WM. Robust relationship inference in genome-wide association studies. Bioinformatics. 2010;26(22):2867-73.

17. Waterboer T, Sehr P, Michael KM, Franceschi S, Nieland JD, Joos TO, et al. Multiplex human papillomavirus serology based on in situ-purified glutathione s-transferase fusion proteins. Clin Chem. 2005;51(10):1845-53.

18. Waterboer T, Sehr P, Pawlita M. Suppression of non-specific binding in serological Luminex assays. J Immunol Methods. 2006;309(1-2):200-4.

19. Kreimer AR, Johansson M, Yanik EL, Katki HA, Check DP, Lang Kuhs KA, et al. Kinetics of the human papillomavirus type 16 E6 antibody response prior to oropharyngeal cancer. J Natl Cancer Inst. 2017;109(8):1-9.

20. Chang CC, Chow CC, Tellier LC, Vattikuti S, Purcell SM, Lee JJ. Secondgeneration PLINK: rising to the challenge of larger and richer datasets. Gigascience. 2015;4:7.

21. Peterson RA, Cavanaugh JE. Ordered quantile normalization: a semiparametric transformation built for the cross-validation era. J Appl Stat. 2019;47(13-15):2312-27.

22. Rentzsch P, Witten D, Cooper GM, Shendure J, Kircher M. CADD: predicting the deleteriousness of variants throughout the human genome. Nucleic Acids Res. 2019:47(D1):D886-D94.

23. Dong S, Boyle AP. Predicting functional variants in enhancer and promoter elements using RegulomeDB. Hum Mutat. 2019;40(9):1292-8.

24. Schmiedel BJ, Singh D, Madrigal A, Valdovino-Gonzalez AG, White BM, Zapardiel-Gonzalo J, et al. Impact of genetic polymorphisms on human immune cell gene expression. Cell. 2018;175(6):1701-15 e16.

25. Sun BB, Maranville JC, Peters JE, Stacey D, Staley JR, Blackshaw J, et al. Genomic atlas of the human plasma proteome. Nature. 2018;558(7708):73-9.

26. Yao C, Chen G, Song C, Keefe J, Mendelson M, Huan T, et al. Genome-wide mapping of plasma protein QTLs identifies putatively causal genes and pathways for cardiovascular disease. Nat Commun. 2018;9(1):3268.

27. Rashkin, S.R., Graff, R.E., Kachuri, L. et al. Pan-cancer study detects genetic risk variants and shared genetic basis in two large cohorts. Nat Commun. 2020;11:4423. https://doi.org/10.1038/s41467-020-18246-6.

28. Lam M, Chen CY, Li Z, Martin AR, Bryois J, Ma X, et al. Comparative genetic architectures of schizophrenia in East Asian and European populations. Nat Genet. 2019;51(12):1670-8.

29. Jun G, Ibrahim-Verbaas CA, Vronskaya M, Lambert JC, Chung J, Naj AC, et al. A novel Alzheimer disease locus located near the gene encoding tau protein. Mol Psychiatry. 2016;21(1):108-17.

30. Motyer A, Vukcevic D, Dilthey A, Donnelly P, McVean G, Leslie S. Practical Use of Methods for Imputation of HLA Alleles from SNP Genotype Data. bioRxiv. 2016:091009. https://doi.org/10.1101/091009.

31. Jia X, Han B, Onengut-Gumuscu S, Chen WM, Concannon PJ, Rich SS, et al. Imputing amino acid polymorphisms in human leukocyte antigens. PLoS One. 2013;8(6):e64683.

32. Moutsianas L, Gutierrez-Achury J. Genetic Association in the HLA Region. In: Evangelou E, editor. Genetic Epidemiology: Methods and Protocols. Methods in Molecular Biology. 1793. 1 ed: Humana Press; 2018. p. 111-34.

33. Purcell S, Neale B, Todd-Brown K, Thomas L, Ferreira MA, Bender D, et al. PLINK: a tool set for whole-genome association and population-based linkage analyses. Am J Hum Genet. 2007;81(3):559-75.

34. Gonzalez-Galarza FF, McCabe A, Santos E, Jones J, Takeshita L, Ortega-Rivera ND, et al. Allele frequency net database (AFND) 2020 update: gold-standard data classification, open access genotype data and new query tools. Nucleic Acids Res. 2020;48(D1):D783-D8.

35. Barbeira AN, Dickinson SP, Bonazzola R, Zheng J, Wheeler HE, Torres JM, et al. Exploring the phenotypic consequences of tissue specific gene 
expression variation inferred from GWAS summary statistics. Nat Commun 2018;9(1):1825

36. Barbeira AN, Bonazzola R, Gamazon ER, Liang Y, Park Y, Kim-Hellmuth S, et al. Widespread dose-dependent effects of RNA expression and splicing on complex diseases and traits. bioRxiv. 2019;814350. https://doi.org/10.1101/814350.

37. PredictDB. MASHR-based gene expression prediction models based on GTEx v8. [Data set]. Zenodo, 2019. https://zenodo.org/record/3518299. Accessed 4 Feb 2020.

38. Urbut SM, Wang G, Carbonetto P, Stephens M. Flexible statistical methods for estimating and testing effects in genomic studies with multiple conditions. Nat Genet. 2019;51(1):187-95.

39. Wen $X$, Lee $Y$, Luca F, Pique-Regi R. Efficient integrative multi-SNP association analysis via deterministic approximation of posteriors. Am J Hum Genet. 2016;98(6):1114-29.

40. Lee Y, Luca F, Pique-Regi R, Wen X. Bayesian Multi-SNP Genetic Association Analysis: Control of FDR and Use of Summary Statistics. bioRxiv. 2018:316471. https://doi.org/10.1101/316471.

41. Steiner I, Kennedy PG, Pachner AR. The neurotropic herpes viruses: herpes simplex and varicella-zoster. Lancet Neurol. 2007;6(11):1015-28.

42. Khalili K, Del Valle L, Otte J, Weaver M, Gordon J. Human neurotropic polyomavirus, JCV, and its role in carcinogenesis. Oncogene. 2003;22(33):5181-91.

43. Feng $H$, Shuda M, Chang Y, Moore PS. Clonal integration of a polyomavirus in human Merkel cell carcinoma. Science. 2008;319(5866):1096-100.

44. Yu G, Wang LG, Han Y, He QY. clusterProfiler: an R package for comparing biological themes among gene clusters. OMICS. 2012;16(5):284-7.

45. Shin OS, Uddin T, Citorik R, Wang JP, Della Pelle P, Kradin RL, et al. LPLUNC1 modulates innate immune responses to Vibrio cholerae. J Infect Dis. 2011; 204(9):1349-57.

46. Shafi O. Inverse relationship between Alzheimer's disease and cancer, and other factors contributing to Alzheimer's disease: a systematic review. BMC Neurol. 2016;16(1):236

47. Bulik-Sullivan B, Finucane HK, Anttila V, Gusev A, Day FR, Loh PR, et al. An atlas of genetic correlations across human diseases and traits. Nat Genet. 2015;47(11):1236-41.

48. Gragert L, Madbouly A, Freeman J, Maiers M. Six-locus high resolution HLA haplotype frequencies derived from mixed-resolution DNA typing for the entire US donor registry. Hum Immunol. 2013;74(10):1313-20.

49. Degenhardt F, Wendorff M, Wittig M, Ellinghaus E, Datta LW, Schembri J, et al. Construction and benchmarking of a multi-ethnic reference panel for the imputation of HLA class I and II alleles. Hum Mol Genet. 2019;28(12):2078-92.

50. Chen Q, Sun L, Chen ZJ. Regulation and function of the CGAS-STING pathway of cytosolic DNA sensing. Nat Immunol. 2016;17(10):1142-9.

51. Kwon J, Bakhoum SF. The cytosolic DNA-sensing CGAS-STING pathway in Cancer. Cancer Discov. 2020;10(1):26-39.

52. Sun L, Wu J, Du F, Chen X, Chen ZJ. Cyclic GMP-AMP synthase is a cytosolic DNA sensor that activates the type I interferon pathway. Science. 2013; 339(6121):786-91.

53. Inoue T, Tsai B. How viruses use the endoplasmic reticulum for entry, replication, and assembly. Cold Spring Harb Perspect Biol. 2013;5(1):a013250.

54. Woo SR, Fuertes MB, Corrales L, Spranger S, Furdyna MJ, Leung MY, et al. STING-dependent cytosolic DNA sensing mediates innate immune recognition of immunogenic tumors. Immunity. 2014;41(5):830-42.

55. Demaria O, De Gassart A, Coso S, Gestermann N, Di Domizio J, Flatz L, et al. STING activation of tumor endothelial cells initiates spontaneous and therapeutic antitumor immunity. Proc Natl Acad Sci U S A. 2015;112(50):15408-13.

56. Ohkuri T, Ghosh A, Kosaka A, Zhu J, Ikeura M, David M, et al. STING contributes to antiglioma immunity via triggering type I IFN signals in the tumor microenvironment. Cancer Immunol Res. 2014;2(12):1199-208.

57. Fu J, Kanne DB, Leong M, Glickman LH, McWhirter SM, Lemmens E, et al. STING agonist formulated cancer vaccines can cure established tumors resistant to PD-1 blockade. Sci Transl Med. 2015:7(283):283ra52.

58. Corrales L, Glickman LH, McWhirter SM, Kanne DB, Sivick KE, Katibah GE, et al. Direct activation of STING in the tumor microenvironment leads to potent and systemic tumor regression and immunity. Cell Rep. 2015;11(7): 1018-30.

59. Ohkuri T, Ghosh A, Kosaka A, Sarkar SN, Okada H. Protective role of STING against gliomagenesis: rational use of STING agonist in anti-glioma immunotherapy. Oncoimmunology. 2015;4(4):e999523.

60. Ikeda K, Nakano R, Uraoka M, Nakagawa Y, Koide M, Katsume A, et al. Identification of ARIA regulating endothelial apoptosis and angiogenesis by modulating proteasomal degradation of CIAP-1 and CIAP-2. Proc Natl Acad Sci U S A. 2009;106(20):8227-32.

61. Verma A, Bhattacharya R, Remadevi I, Li K, Pramanik K, Samant GV, et al. Endothelial cell-specific chemotaxis receptor (ecscr) promotes angioblast migration during vasculogenesis and enhances VEGF receptor sensitivity. Blood. 2010;115(22):4614-22.

62. Astle WJ, Elding $H$, Jiang $T$, Allen D, Ruklisa D, Mann AL, et al. The allelic landscape of human blood cell trait variation and links to common complex disease. Cell. 2016;167(5):1415-29 e19.

63. Assinger A. Platelets and infection - an emerging role of platelets in viral infection. Front Immunol. 2014:5:649.

64. Kelly RJ, Rouquier S, Giorgi D, Lennon GG, Lowe JB. Sequence and expression of a candidate for the human secretor blood group alpha $(1,2)$ fucosyltransferase gene (FUT2). Homozygosity for an enzyme-inactivating nonsense mutation commonly correlates with the non-secretor phenotype. J Biol Chem. 1995;270(9):4640-9.

65. Hazra A, Kraft P, Selhub J, Giovannucci EL, Thomas G, Hoover RN, et al. Common variants of FUT2 are associated with plasma vitamin B12 levels. Nat Genet. 2008;40(10):1160-2.

66. Carlsson B, Kindberg E, Buesa J, Rydell GE, Lidon MF, Montava R, et al. The G428A nonsense mutation in FUT2 provides strong but not absolute protection against symptomatic Gll.4 Norovirus infection. PLoS One. 2009; 4(5):e5593.

67. Ruvoen-Clouet N, Belliot G, Le Pendu J. Noroviruses and histo-blood groups: the impact of common host genetic polymorphisms on virus transmission and evolution. Rev Med Virol. 2013;23(6):355-66.

68. Imbert-Marcille BM, Barbe L, Dupe M, Le Moullac-Vaidye B, Besse B, Peltier C, et al. A FUT2 gene common polymorphism determines resistance to rotavirus A of the P [8] genotype. J Infect Dis. 2014;209(8):1227-30.

69. Ikehara Y, Nishihara S, Yasutomi H, Kitamura T, Matsuo K, Shimizu N, et al. Polymorphisms of two fucosyltransferase genes (Lewis and Secretor genes) involving type I Lewis antigens are associated with the presence of anti-Helicobacter pylori lgG antibody. Cancer Epidemiol Biomark Prev. 2001;10(9):971-7.

70. Blackwell CC, Jonsdottir K, Hanson M, Todd WT, Chaudhuri AK, Mathew B, et al. Non-secretion of $\mathrm{ABO}$ antigens predisposing to infection by Neisseria meningitidis and Streptococcus pneumoniae. Lancet. 1986;2(8501):284-5.

71. de Lange KM, Moutsianas L, Lee JC, Lamb CA, Luo Y, Kennedy NA, et al. Genome-wide association study implicates immune activation of multiple integrin genes in inflammatory bowel disease. Nat Genet. 2017:49(2):256-61.

72. Ellinghaus D, Jostins L, Spain SL, Cortes A, Bethune J, Han B, et al. Analysis of five chronic inflammatory diseases identifies 27 new associations and highlights disease-specific patterns at shared loci. Nat Genet. 2016;48(5):510-8.

73. Hoffmann TJ, Theusch E, Haldar T, Ranatunga DK, Jorgenson E, Medina MW, et al. A large electronic-health-record-based genome-wide study of serum lipids. Nat Genet. 2018;50(3):401-13.

74. Tanaka T, Scheet P, Giusti B, Bandinelli S, Piras MG, Usala G, et al. Genomewide association study of vitamin B6, vitamin B12, folate, and homocysteine blood concentrations. Am J Hum Genet. 2009;84(4):477-82.

75. Liu M, Jiang Y, Wedow R, Li Y, Brazel DM, Chen F, et al. Association studies of up to 1.2 million individuals yield new insights into the genetic etiology of tobacco and alcohol use. Nat Genet. 2019;51(2):237-44.

76. McKay JD, Hung RJ, Han Y, Zong X, Carreras-Torres R, Christiani DC, et al. Large-scale association analysis identifies new lung cancer susceptibility loci and heterogeneity in genetic susceptibility across histological subtypes. Nat Genet. 2017;49(7):1126-32.

77. Batista CM, Mariano ED, Barbosa BJ, Morgalla M, Marie SK, Teixeira MJ, et al. Adult neurogenesis and glial oncogenesis: when the process fails. Biomed Res Int. 2014:2014:438639.

78. Yamagishi S, Yamada K, Sawada M, Nakano S, Mori N, Sawamoto K, et al. Netrin-5 is highly expressed in neurogenic regions of the adult brain. Front Cell Neurosci. 2015:9:146.

79. Leong YA, Chen Y, Ong HS, Wu D, Man K, Deleage C, et al. CXCR5(+) follicular cytotoxic T cells control viral infection in B cell follicles. Nat Immunol. 2016;17(10):1187-96.

80. Willis TG, Zalcberg IR, Coignet LJ, Wlodarska I, Stul M, Jadayel DM, et al. Molecular cloning of translocation $t(1 ; 14)(q 21 ; q 32)$ defines a novel gene (BCL9) at chromosome 1q21. Blood. 1998;91(6):1873-81.

81. Deka J, Wiedemann N, Anderle P, Murphy-Seiler F, Bultinck J, Eyckerman S, et al. $\mathrm{BC}|9 / \mathrm{BC}| 9 \mid$ are critical for Wnt-mediated regulation of stem cell traits in colon epithelium and adenocarcinomas. Cancer Res. 2010;70(16):6619-28. 
82. Pilli M, Arko-Mensah J, Ponpuak M, Roberts E, Master S, Mandell MA, et al. TBK-1 promotes autophagy-mediated antimicrobial defense by controlling autophagosome maturation. Immunity. 2012;37(2):223-34.

83. Gordon DE, Jang GM, Bouhaddou M, Xu J, Obernier K, White KM, et al. A SARS-CoV-2 protein interaction map reveals targets for drug repurposing. Nature. 2020:583:459-68. https://doi.org/10.1038/s41586-020-2286-9.

84. Zhu L, Li Y, Xie X, Zhou X, Gu M, Jie Z, et al. TBKBP1 and TBK1 form a growth factor signalling axis mediating immunosuppression and tumourigenesis. Nat Cell Biol. 2019;21(12):1604-14.

85. Yang J, Wang B, Chen H, Chen X, Li J, Chen Y, et al. Thyrotroph embryonic factor is downregulated in bladder cancer and suppresses proliferation and tumorigenesis via the AKT/FOXOs signalling pathway. Cell Prolif. 2019;52(2): e12560.

86. International Multiple Sclerosis Genetics C, Wellcome Trust Case Control C, Sawcer S, Hellenthal G, Pirinen M, Spencer CC, et al. Genetic risk and a primary role for cell-mediated immune mechanisms in multiple sclerosis. Nature. 2011;476(7359):214-9.

87. Sun MY, Wang Y, Zhu J, Lv C, Wu K, Wang XW, et al. Critical role for nonGAP function of Galphas in RGS1mediated promotion of melanoma progression through AKT and ERK phosphorylation. Oncol Rep. 2018;39(6): 2673-80.

88. Carreras J, Kikuti YY, Bea S, Miyaoka M, Hiraiwa S, Ikoma H, et al. Clinicopathological characteristics and genomic profile of primary sinonasal tract diffuse large $B$ cell lymphoma (DLBCL) reveals gain at 1q31 and RGS1 encoding protein; high RGS1 immunohistochemical expression associates with poor overall survival in DLBCL not otherwise specified (NOS). Histopathology. 2017;70(4):595-621

89. Mukherjee S, Mez J, Trittschuh EH, Saykin AJ, Gibbons LE, Fardo DW, et al. Genetic data and cognitively defined late-onset Alzheimer's disease subgroups. Mol Psychiatry. 2018. https://doi.org/10.1038/s41380-018-0298-8.

90. Keren-Shaul H, Spinrad A, Weiner A, Matcovitch-Natan O, Dvir-Szternfeld R, Ulland TK, et al. A unique microglia type associated with restricting development of Alzheimer's disease. Cell. 2017;169(7):1276-90 e17.

91. Jawaheer D, Li W, Graham RR, Chen W, Damle A, Xiao X, et al. Dissecting the genetic complexity of the association between human leukocyte antigens and rheumatoid arthritis. Am J Hum Genet. 2002;71(3):585-94.

92. Vader W, Stepniak D, Kooy Y, Mearin L, Thompson A, van Rood JJ, et al. The HLA-DQ2 gene dose effect in celiac disease is directly related to the magnitude and breadth of gluten-specific T cell responses. Proc Natl Acad Sci U S A. 2003;100(21):12390-5.

93. Erlich H, Valdes AM, Noble J, Carlson JA, Varney M, Concannon P, et al. HLA DR-DQ haplotypes and genotypes and type 1 diabetes risk: analysis of the type 1 diabetes genetics consortium families. Diabetes. 2008;57(4):1084-92.

94. Raychaudhuri S, Sandor C, Stahl EA, Freudenberg J, Lee HS, Jia X, et al. Five amino acids in three HLA proteins explain most of the association between MHC and seropositive rheumatoid arthritis. Nat Genet. 2012:44(3):291-6.

95. Hu X, Deutsch AJ, Lenz TL, Onengut-Gumuscu S, Han B, Chen WM, et al. Additive and interaction effects at three amino acid positions in HLA-DQ and HLA-DR molecules drive type 1 diabetes risk. Nat Genet. 2015;47(8): 898-905.

96. Patsopoulos NA, Barcellos LF, Hintzen RQ, Schaefer C, van Duijn CM, Noble $J A$, et al. Fine-mapping the genetic association of the major histocompatibility complex in multiple sclerosis: HLA and non-HLA effects. PLoS Genet. 2013;9(11):e1003926.

97. Paulson KG, Tegeder A, Willmes C, Iyer JG, Afanasiev OK, Schrama D, et al. Downregulation of $\mathrm{MHC}-\mathrm{I}$ expression is prevalent but reversible in Merkel cell carcinoma. Cancer Immunol Res. 2014;2(11):1071-9.

98. Candore G, Lio D, Colonna Romano G, Caruso C. Pathogenesis of autoimmune diseases associated with 8.1 ancestral haplotype: effect of multiple gene interactions. Autoimmun Rev. 2002;1(1-2):29-35.

99. Ferreiro-Iglesias A, Lesseur C, McKay J, Hung RJ, Han Y, Zong X, et al. Fine mapping of $\mathrm{MHC}$ region in lung cancer highlights independent susceptibility loci by ethnicity. Nat Commun. 2018;9(1):3927

100. Abdou AM, Gao X, Cozen W, Cerhan JR, Rothman N, Martin MP, et al. Human leukocyte antigen (HLA) A1-B8-DR3 (8.1) haplotype, tumor necrosis factor (TNF) G-308A, and risk of non-Hodgkin lymphoma. Leukemia. 2010; 24(5):1055-8.

101. Sundqvist E, Buck D, Warnke C, Albrecht E, Gieger C, Khademi M, et al. JC polyomavirus infection is strongly controlled by human leucocyte antigen class II variants. PLoS Pathog. 2014;10(4):e1004084.
102. Khandaker GM, Cousins L, Deakin J, Lennox BR, Yolken R, Jones PB. Inflammation and immunity in schizophrenia: implications for pathophysiology and treatment. Lancet Psychiatry. 2015;2(3):258-70.

103. Dickerson F, Jones-Brando L, Ford G, Genovese G, Stallings C, Origoni A, et al. Schizophrenia is associated with an aberrant immune response to Epstein-Barr virus. Schizophr Bull. 2019;45(5):1112-9.

104. International Schizophrenia C, Purcell SM, Wray NR, Stone JL, Visscher PM, O'Donovan MC, et al. Common polygenic variation contributes to risk of schizophrenia and bipolar disorder. Nature. 2009;460(7256):748-52.

105. Schizophrenia Working Group of the Psychiatric Genomics C. Biological insights from 108 schizophrenia-associated genetic loci. Nature. 2014; 511(7510):421-7.

106. Bronson PG, Chang D, Bhangale T, Seldin MF, Ortmann W, Ferreira RC, et al. Common variants at PVT1, ATG13-AMBRA1, AHI1 and CLEC16A are associated with selective IgA deficiency. Nat Genet. 2016;48(11):1425-9.

107. Sekar A, Bialas AR, de Rivera H, Davis A, Hammond TR, Kamitaki N, et al. Schizophrenia risk from complex variation of complement component 4. Nature. 2016:530(7589):177-83.

108. Fry A, Littlejohns TJ, Sudlow C, Doherty N, Adamska L, Sprosen T, et al. Comparison of Sociodemographic and health-related characteristics of UK biobank participants with those of the general population. Am J Epidemiol. 2017;186(9):1026-34

\section{Publisher's Note}

Springer Nature remains neutral with regard to jurisdictional claims in published maps and institutional affiliations.
Ready to submit your research? Choose BMC and benefit from:

- fast, convenient online submission

- thorough peer review by experienced researchers in your field

- rapid publication on acceptance

- support for research data, including large and complex data types

- gold Open Access which fosters wider collaboration and increased citations

- maximum visibility for your research: over $100 \mathrm{M}$ website views per year

At BMC, research is always in progress.

Learn more biomedcentral.com/submissions 